\title{
Ages and Metallicities of Early-Type Void Galaxies from Line Strength Measurements
}

\author{
Gary Wegner 1 , Norman A. Grogin 20
}

\begin{abstract}
We present spectroscopic observations of 26 galaxies of type E and S0, based on their blue morphologies, located in voids by the study of Grogin \& Geller (1999). Measurements of redshift, velocity dispersion, and four Lick line indices, $\mathrm{Mg}_{b}, \mathrm{Fe} 5270, \mathrm{Fe} 5335$, and $\mathrm{H} \beta$ with their errors are given for all of these galaxies, along with $\mathrm{H} \beta$, [OIII], $\mathrm{H} \alpha$, and [NII] emission line strengths for a subset of these objects. These sources are brighter than $M^{*}$ for low density regions and tend to be bluer than their counterpart early-type objects in high density regions. Using the models of Thomas et al. (2003) gives metal abundances and ages with a median $\alpha$ enhancement, $[\alpha / F e]=+0.13$, and median metals abundance, $[Z / H]=+0.22$, values comparable to those found for $\mathrm{E}$ and $\mathrm{S} 0$ galaxies in clusters, but with a wider spread in $[\mathrm{Z} / \mathrm{H}]$ towards low values. If the emission line subsample is interpreted as younger, the proportion of young objects is higher than for earlytypes in higher density regions. There is a significant incidence of sources in the sample with emission lines in their spectra ( $46 \%$ with $\mathrm{H} \beta$ and [OIII] and $69 \%$ with $\mathrm{H} \alpha$ or $[\mathrm{NII}])$ as well as shells and rings in their morphologies $(19 \%)$. The diagnostic $\log [\mathrm{NII}] / \mathrm{H} \alpha, \log [\mathrm{OIII}] / \mathrm{H} \beta$ diagram places 10 of 12 emission line galaxies in or near the star forming and liner region and two among the Seyferts. The $\mathrm{H} \alpha$ fluxes indicate star formation rates of 0.2 to $1.0 M_{\odot} \mathrm{yr}^{-1}$. The percentage of these early-type void galaxies undergoing star formation appears to be higher compared to their cluster counterparts and the range of ages wider.
\end{abstract}

Subject headings: cosmology: large-scale structure of the universe - galaxies: distances and redshifts - galaxies: elliptical and lenticular, $\mathrm{cD}$ - galaxies: general — galaxies: Stellar content — techniques: spectroscopic

\footnotetext{
${ }^{1}$ Department of Physics \& Astronomy, Dartmouth College, 6127 Wilder Laboratory, Hanover, NH 03755; gaw@bellz.dartmouth.edu

${ }^{2}$ School of Earth and Space Exploration, Arizona State University, PO Box 871404, Tempe, AZ 852871404; nagrogin@asu.edu
} 


\section{INTRODUCTION}

Less has been known about early-type galaxies in low density environments than those in the higher density regions of galaxy clusters outlining large-scale structure. Cosmological data (e.g., Spergel et al.2003; Efstathiou et al.2002) and many recent observations of galaxies and galaxy clusters indicate that galaxy evolution follows a complicated tree of mergers (e.g., Cole et al. 2000; Benson et al. 2001). Galaxy color indices and line strengths have been brought to bear on these questions using increasingly complicated models (e.g., Tinsley 1978; Bruzual \& Charlot 1993; Worthey 1994; Colless et al. 1999; Nelan et al. 2005). Peebles (2001) and Nusser, Gubser, \& Peebles (2005) discusses problems posed by the void phenomenon, which offer a clue to understanding large-scale structure formation. Recent progress in understanding galaxy evolution has given impetus to considering galaxies in the voids. The question then is, how do the galaxies in the voids differ from those in clusters?

For void galaxies (hereafter VGs), the problem that arises is the construction of a sizable sample of objects definitely in the voids. Consequently there exists a range of definitions of VGs. Grogin \& Geller (1999, hereafter GG99) located galaxies within three nearby voids using a $5 h^{-1} \mathrm{Mpc}$-smoothed density estimator and the CfA2 redshift survey (Geller \& Huchra 1989) of the Updated Zwicky Catalog (UZC, Falco et al. 1989). In general, GG99 concluded what is now recognized by many studies, viz. compared to galaxies in higher density regions, the $(B-R)$ color distribution of VGs is significantly bluer and the most luminous galaxies $\left(M_{R} \leq-21\right.$ mag.) are absent. Grogin \& Geller (2000; hereafter GG00) noted that while galaxy morphologies vary little with density, there is a reduction in the frequency of early-types and and an increase of irr/pec types in the lower density environments. G00 also found that neighbors in the voids have 1) sharply reduced velocity separations and 2) much higher incidence of prolific star formation as compared to near-neighbors outside voids. Patiri et al. (2006) find similar results from 495 SDSS galaxies.

Numerous investigations have studied galaxy properties in different density environments. Bernardi et al. (1998) broke the ENEAR early-type galaxies into those belonging to clusters and those in the field. They used the $\mathrm{Mg}_{b}$ index and concluded that most of the stars

originated at $z>3$ with no difference between high and low density regions. Kuntschner et al. (2002) studied 9 early-type galaxies in low density environments and concluded that their formation continued to $z \leq 1$ while in clusters it ended by $z \geq 2$. Colbert et al. (2001) found 30 isolated early-type galaxies using the RC3 (de Vaucouleurs et al. 1991). Thomas et al. (2005) compared 54 early-type galaxies with 70 in low density regions, finding evidence for the influence of environment on the stellar populations. Their massive objects in low density regions appeared about 2 Gyr younger and marginally more metal rich with no significant $[\alpha / F e]$ differences. Colobert et al. (2006) have measured Lick indices for 22 objects in low 
density regions, concluding that they show a wider range in metals and ages than cluster galaxies. Sil'chenko (2006) studied nearby S0s in different environments and finds objects in sparse environments tend to be younger than those in dense regions.

The number of objects used in statistical studies has been steadily increasing. Rojas et al. (2005) used 1010 galaxies in voids, but these are not sorted by their morphological types. Bernardi et al. (2003a,b) studied the properties of $\sim 9000$ early-type galaxies using spectroscopic data from the Sloan Digital Sky Survey (SDSS); although the Fundamental Plane of early-types in dense and sparse regions may vary slightly, the spectra and colormagnitude relations were not seen to differ significantly. Bernardi et al. (2006) extended studies to 39320 early-type galaxies in different environments finding that objects in low density regions have had the more recent star formation. Kauffmann et al. (2006) studied star formation in 46892 SDSS galaxies in different environments and determine that the most sensitive environmental differences occur for objects with stellar mass $M_{\star}<3 \times 10^{10} M_{\odot}$. One should note that although numerous, the SDSS spectra are fiber-fed and thus sample only the inner regions of nearby galaxies; even as far as $\sim 150 \mathrm{Mpc}$, a $3^{\prime \prime}$ diameter aperture corresponds to $\sim 2 \mathrm{kpc}$ on the object.

Similar data are becoming available for high redshift galaxies (e.g., Faber et al.2005; Glazebrook et al.2004); these latter authors conclude that there is evidence that galaxy formation has continued up to the present and that at least $67 \%$ by mass have formed since $z \approx 2$. Schiavon et al. (2006) find ages in red field galaxies are inconsistent with all their stars having formed at high redshift and then passively evolving.

Other investigators studied galaxies in different density regions of clusters. Poggianti et al. (2001a,b) analyzed Lick indices for Coma galaxies finding trends with both galaxy luminosity and environment. Carter et al. (2002) saw a significant $M g_{2}$ radial gradient in Coma, but only weak $\langle F e>$ and $\mathrm{H} \beta$ gradients. Edwards et al. (2002) studied giant and dwarf galaxies in Coma, obtained evidence for differences in dynamics, and concluded that the Coma cluster was formed by mergers. Mehlert et al. (2003) also surmised that mergers of galaxies occurred from the radial dependence of line strengths in individual Coma galaxies. Ogando et al. (2005) utilized the $\mathrm{Mg}_{2}$ radial gradients in a much larger field sample and inferred that both the monolithic dissipative collapse and hierarchical merging processes can contribute to the buildup of elliptical galaxies. Nelan et al. (2005) studied 4097 red sequence galaxies in 93 low $z$ clusters using Lick indices and velocity dispersions and found evidence for evolutionary effects including 'downsizing' (i.e. stars in the most massive ellipticals have existed on the red sequence since early epochs, but most lower mass ellipticals have only recently reached the red sequence). Smith et al. $(2004,2006)$ utilized this same galaxy sample and found radial age and $[\alpha / \mathrm{Fe}]$ gradients within galaxy clusters, suggesting that 
early-type galaxies in the cluster cores were accreted earlier than those at the outer radii. Rines et al. (2005) have used $\mathrm{H} \alpha$ emission to estimate star formation in cluster galaxies as a function of environment and found that the kinematics of star forming galaxies in the infall regions of clusters rule out some 'splash back' mechanisms.

Whilst earlier semi-analytical models of hierarchical galaxy formation had problems matching the observed properties of elliptical galaxies including 'downsizing,' more recent models are more successful. Tantalo \& Chiosi (2002) used N-body Tree-SPH models of elliptical galaxies in normal environments. They found that the hierarchical scenario enhances $[\alpha / \mathrm{Fe}]$ over a wide range of ages. De Lucia et al. (2005) studied the evolutionary histories of ellipticals in the hierarchical model using large high-resolution simulations combined withe a semi-analytic model in the concordance $\Lambda$ CDM cosmology. They predict that the average ages, metallicites, and redness of elliptical galaxies increase with the density of their environment and achieve better agreement with the observations than older models. Utilizing models that include the AGN feedback mechanism of Croton et al. (2006) which suppresses gas condensation by cooling flows, they found that star formation peaks near $z=5$, but lasts longer for field ellipticals than for the cluster ellipticals where it is more sharply peaked for galaxies of mass $10^{12} \mathrm{M}_{\odot}$ than for ones of $10^{9} \mathrm{M}_{\odot}$. Hopkins et al. (2006) compute merger models which include super massive black holes and match well several of the existing data for early-type galaxies. Khochfar \& Silk (2006) argue that stars in ellipticals and bulges form from two main components, initially from mergers which are then added to later by quiescent gaseous disks formed from mergers.

In this paper we report spectroscopic observations of the Lick system $\mathrm{Mg}, \mathrm{Fe}$, and $\mathrm{H} \beta$ line strengths as well as emission line strengths for 26 galaxies located in nearby voids selected using the rigorous method of GG99 and GG00. §2 presents the sample selection and its basic properties. $\S 3$ describes the observations and gives the line strength measurements. $\S 4$ uses the line indices to determine metal abundances and ages. $\S 5$ compares these data with galaxies in denser regions and models and determines star formation rates. $\S 6$ presents our conclusions and discusses them in light of current ideas on galaxy formation. Throughout this paper we assume $H_{0}=70 \mathrm{~km} \mathrm{~s}^{-1}, \Omega_{M}=0.3$, and $\Omega_{\Lambda}=0.7$.

\section{SAMPLE DEFINITION}

\subsection{Void Locations}

Figures 1 and 2 give the locations of the 26 galaxies in the present study. They are a subset of the VGs defined in the GG99 sample, where the large-scale galaxy number 
density was estimated by smoothing the CfA2 galaxy distribution with a $5 h^{-1}$ Mpc Gaussian kernel normalized by the CfA2 selection function. From this large-scale density map, GG99 targeted 280 galaxies within and bordering three prominent "voids" in CfA2 at $c z \sim 5000$ $10000 \mathrm{~km} \mathrm{~s}^{-1}$ (see Fig. 1 of GG99). Fainter galaxies in the same regions as found by the narrower but deeper Century Survey ( $R \lesssim 16.1$, Geller et al. 1997; Wegner et al. 2001) and 15R Survey ( $R \lesssim 15.4$, Geller et al., in prep.) were also included (see Figs. 1 and 2 of GG99) in order to investigate lower-luminosity VGs below the UZC flux limit $(B \lesssim 15.5)$.

We selected early-type VGs from the GG99 sample according to the $B$-band morphological classifications (GG00) of the GG99 imaging, taken with $\approx 1$ 1.5-2'.0 seeing. We secured spectroscopic observations for 26 of the 35 galaxies in GG99 located in under dense regions (density contrast $n / \bar{n}<1$ ) and with revised Hubble type $T \leq-2$ (S0 or earlier), where $n$ is the smoothed galaxy number density at a point $(\alpha, \delta, z)$ and $\bar{n}$ is the mean density for the CfA2 survey as defined in GG99.

The 26 spectroscopically observed galaxies are listed in Table 1. Column (1) is an ordinal reference number, and column (2) is the redshift-catalog designation. Columns (3) and (4) list the J2000 right ascension and declination, respectively. Column (5) is the redshift from GG99 and GG00 corrected to the local standard of rest using $300 \sin 1$ cos b km s${ }^{-1}$. Column

(6) gives new morphological types estimated using the blue copies of the POSS2 described in $§ 2.2$. Finally, Columns (7), (8), and (9) give the $B, R$, and $K$-band absolute magnitudes. The $B$ and $R$ data are described in GG99 and GG00 and all the VGs were observed by 2Mass (Skrutskie et al.2006). We used the total apparent magnitudes and the redshifts to calculate the absolute magnitudes corrected for galactic absorption using Schlegel et al. (1998) in NED.

\subsection{Morphologies and Luminosities}

Histograms of the absolute magnitudes of the 26 observed VGs are given in Figure 3. Comparing to luminosity functions of nearby galaxy clusters e.g., Virgo and Fornax (e.g., Jerjen \& Tammann 1997) or more recent redshift survey results shows that these VGs are at the bright end of the luminosity function for ellipticals. They have an average absolute magnitude $\bar{M}_{B}=-18.7, \bar{M}_{R}=-20.4$, and $\bar{M}_{K}=-23.5$. Thus most of our VGs are brighter than $M_{b j}^{*} \approx-17.8$ found for early-type void galaxies by Croton et al. (2005) or $M_{r}^{*} \approx-19.0$ (Hoyle et al. 2005) which is roughly a magnitude fainter than for objects in higher density regions..

The color-magnitude diagram for the VGs is also shown in Figure 3. For comparison, 
the boundaries of the Coma cluster red sequence estimated from Mobasher et al. (2001) assuming a distance modulus, $D M=35.0$ mag. are given. The present VGs mostly lie near the Coma red sequence, but four objects definitely lie below (i.e. are bluer) of which three have the $\mathrm{H} \beta$ emission discussed in $\S 3.2 .2$. The average $(B-R)$ of those with emission is $(B-R)=1.3$ compared to 1.4 without emission respectively.

Figure 4 presents $2^{\prime} \times 2^{\prime}$ pictures of the 26 observed VGs taken from the digitized blue POSS-II 1. Blue images have the advantage that they are more sensitive to spiral structure and star forming regions. Although most of these objects appear as early-type (E or S0) galaxies, five or $19 \%$ show evidence of structure e.g., rings or shells and deep imaging may increase this proportion. Colbert et al. (2001) also examined morphologies of 30 isolated early-type galaxies using deep imaging and found a $41 \%$ incidence of shells or tidal features compared to $8 \%$ for a group sample.

\section{OBSERVATIONS}

\subsection{Spectroscopic Data}

Long slit spectra of the 26 VGs were observed 1999 November and 2000 May, at the MDM Observatory on Kitt Peak using the MODSPEC spectrograph attached to the $2.4 \mathrm{~m}$ Hiltner telescope. Additional observations of 14 VGs were secured in 2003 November and 2004 April with the same instrumental setup. The detector was a $2048^{2}$ Site device covering

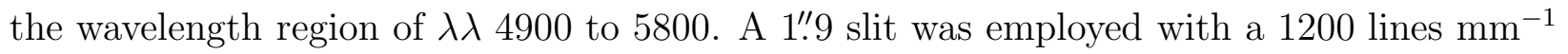
grating (original dispersion of $1.01 \AA \AA^{-1} \mathrm{pix}^{-1}$ ) giving $2.0 \AA$ resolution. For all spectra, the slit length exceeded $6^{\prime}$ which can be compared with the $\sim 1^{\prime}$ size of the galaxies. Generally each observation consisted of three 30 or 60 minute integrations which yielded a combined $S / N>60-90$ per pixel near $\lambda$ 5200. The slit ran N-S for the 1999-2000 observations; in 2003-2004 it was along the galaxy's major axis. Three kinds of standards were observed:

\footnotetext{
1 The Digitized Sky Surveys were produced at the Space Telescope Science Institute under U.S. Government grant NAG W-2166. The images of these surveys are based on photographic data obtained using the Oschin Schmidt Telescope on Palomar Mountain and the UK Schmidt Telescope. The plates were processed into the present compressed digital form with the permission of these institutions. The Second Palomar Observatory Sky Survey (POSS-II) was made by the California Institute of Technology with funds from the National Science Foundation, the National Geographic Society, the Sloan Foundation, the Samuel Oschin Foundation, and the Eastman Kodak Corporation. The Oschin Schmidt Telescope is operated by the California Institute of Technology and Palomar Observatory.
} 
spectrophotometric as implemented in IRAF2; radial velocity with spectral class near K III; and Lick Standards from Worthey et al. (1994) and Trager et al. (1998) for line strength calibration. Hg-Ne-Xe comparisons were taken before and after object exposures. Typically about five Lick and radial velocity standards and four flux standards were observed each night.

Additional spectra of 22 of the VGs covering $\lambda \lambda 6260-7175$ and $\lambda \lambda 4250-5150$ were secured in 2006 November using the CCDS spectrograph with a Loral $1200 \times 800$ CCD on the Hiltner telescope. A 600 lines $\mathrm{mm}^{-1}$ grating (original dispersion $0.79 \AA_{\text {Apix }}^{-1}$ ), a LB370 order separation filter, and a 1".7 slit placed N-S were used which yields a $3.0 \AA$ resolution. Two 15 min. exposures were obtained of each galaxy giving $S / N \sim 20-40$ in the continuum near $\lambda 6700$ when summed, but the emission lines were typically a factor $30-100$ stronger. Spectra of eight objects, including the six remaining, were obtained in 2007 March with the same spectrograph but with a 150 lines $\mathrm{mm}^{-1}$ grating (original dispersion $3.29 \AA_{\text {pix }}^{-1}$ ) and a 1".3 slit running E-W yielding $12 \AA$ resolution covering the wavelength range $\lambda \lambda 4000-7200$. Single 15 min. exposures of the galaxies were taken with this setup which yielded $S / N \sim 30$ in the continuum near $\lambda 6700$ but again the emission lines can be many times stronger than the continuum. Calibration spectra and stars were observed as above.

Standard reductions of the spectra used the longslit option in IRAF. This included bias subtraction, flat fielding, and wavelength calibration. For sky subtraction the background routine was employed. The final one dimensional spectra were extracted using apsum with the variance weighting option and including all of the galaxy's light to a point where the luminosity had fallen to $10 \%$ of its peak value.

Figure 5 gives examples of the blue and red portions of the VG spectra. Note that although all have been corrected for the spectral response of the apparatus, the intensity scales are on a relative scale. Galaxies 21 and 22 show the strong emission lines in both the blue and red regions. If these lines are eliminated, the underlying spectra show the typical $\mathrm{Mg}_{b}$ and iron lines although a bluer continuum. Objects 15 and 17 have weaker emission lines in the blue and have absorption lines that are more typical of early-types, but they have strong emission in the red. Galaxies 4 and 24 have emission in the red, primarily weaker [NII] $\lambda 6583$, weak or absent $\mathrm{H} \alpha$ presumably filling in the underlying absorption line, and no detectable blue emissions. Many of other galaxies, e.g., 3, 5, and 18 (not shown) appear as normal early-types with no detectable emission lines. The emission lines are discussed further in $\S 5.2$.

\footnotetext{
${ }^{2}$ IRAF is distributed by the National Optical Astronomy Observatories which is operated by the Association of Universities for Research in Astronomy, Inc. under contract with the National Science Foundation.
} 
The heliocentric redshifts, $c z$, and velocity dispersions, $\sigma$, were measured from the higher resolution 1999 - 2004 spectra as in Wegner et al. (1999, 2003) employing the cross correlation method (Tonry \& Davis 1979) in the IRAF task fxcor. The correlation peak width is calibrated by convolving the standard star spectra with Gaussians of known width to obtain $\sigma$; measurements used rest wavelengths of $\lambda \lambda 4900$ to 5500 in the galaxy spectra.

\subsection{Line Strength Measurements}

\subsubsection{Absorption Line Data}

Line strengths for four Lick system absorption features were obtained for all spectra taken in 1999 - 2004: $\mathrm{H} \beta, \mathrm{Mg}_{b}, \mathrm{Fe} \lambda 5270$, and Fe $\lambda 5335$. The galaxy and standard star spectra were Gaussian convolved to degrade their resolution to the Lick $8.4 \AA$ value and corrected for instrumental spectral sensitivity using spectrophotometric standards. Instrumental indices were placed on the Lick system utilizing the stellar standards and applied to the galaxy data. The line indices are defined in Trager et al. (1998). Figure 6 compares our standard star indices with those published (Worthey et al. (1994); Trager et al. 1998) after correction: the $\mathrm{H} \beta, \mathrm{Mg}_{b}, \mathrm{Fe} \lambda 5270$, and Fe $\lambda 5335$ were multiplied by 0.997, 1.393, 1.284, and 1.862 respectively. Galaxy indices were corrected for the broadening due to $\sigma$. Several authors including Gonzales (1993) and Trager et al. (1998) have published nearly identical corrections for many indices. Here we adopt Gonzales' (1993) values, although we note that these can change for stellar populations not well matched by K giant spectra (Schiavon 2007). The errors in the redshifts, velocity dispersions, and line indices were estimated from the standard deviation of the mean of individual spectra of each galaxy.

\subsubsection{Emission Line Data and $H \beta$ Corrections}

Equivalent widths for the emission lines were determined using the IRAF routine splot. These data are discussed further in $§ 5.2$. Most of these objects have $\mathrm{H} \alpha$ emission measurements that are reported in GG00. On the whole the agreement is good, but with some large differences which we attribute as due to the range in slit widths and position angles used in the different observations and could also affect the simple $\mathrm{H} \alpha$ infill correction used below.

It is well known that $\mathrm{H} \beta$ indices can suffer from emission contamination Many investigators corrected for this emission by subtracting a fraction of the combined equivalent widths of the $\lambda 5007$ and $\lambda 4957$ [OIII] lines. Gonzales (1993) multiplies by 0.7 while Kuntschner et al. (2001) determine 0.6. Nelan et al. (2005) show that this simple correction is subject 
to large errors and often underestimates the $\mathrm{H} \beta$ emission by large factors. However they demonstrate a tight correlation between $\mathrm{H} \alpha$ and $\mathrm{H} \beta$ finding that the $\mathrm{H} \alpha / \mathrm{H} \beta$ ratio is $4.5_{-1.6}^{+1.5}$. This can be compared with Stasińska et al. (2004) who demonstrated a tight relation for spirals and obtained a ratio of 5.4. Here we utilize the 4.5 factor of Nelan et al. to correct $\mathrm{H} \beta$.

$\mathrm{H} \alpha$ must also be adjusted for underlying absorption in the lower resolution spectra shown in Figure 4. The mean of the four strongest $\mathrm{H} \alpha$ absorption line equivalent widths in the VGs which appear to be free of any emission lines is $2.32 \pm 0.22 \AA$. We then compensate for absorption assuming this mean value. We do not make an absorption correction for $\mathrm{H} \beta$ which is observed in the higher resolution spectra and where the line profiles are well resolved. Here negative values of line strengths denote emission features. Therefore the corrections to the equivalent widths of the two lines in $\AA$ were obtained using the following two steps:

1. Correct the equivalent width of $\mathrm{H} \alpha$ for absorption using $\mathrm{H} \alpha^{(\mathrm{em})}=\mathrm{H} \alpha^{(\mathrm{obs})}-2.32$.

2. The emission correction for $\mathrm{H} \beta$ is then obtained using $\operatorname{Corr}(\mathrm{H} \beta)=\mathrm{H} \alpha^{(\mathrm{em})} / 4.5$.

\subsubsection{Line Strength Measurement Results}

Table 2 summarizes the absorption line data for the VGs. Column (1) lists the ID number from Table 1. Column (2) is the number of runs the galaxy was observed. Columns (3) and (4) give our redshift and its error. Columns (5) and (6) are $\sigma$ and its error. Columns

(7) and (8) are the $\mathrm{H} \beta$ index, uncorrected for emission, and its error; column (9) is the correction to $\mathrm{H} \beta$ as derived above Columns (10)-(15) are the $\mathrm{Mg}_{b}$ and iron indices and their errors. No aperture corrections have been applied to the values in the table. Using the correction methods of Jørgensen et al. (1995) and Nelan et al. (2005), these are found to be of order 0.01 .

Table 3 presents the equivalent width measurements of $\mathrm{H} \alpha,[\mathrm{NII}] \lambda 6583, \mathrm{H} \beta$, and [OIII $] \lambda 5007$. Column (1) is the ID number, Columns (2) and (3) give the uncorrected equivalent widths of the first two lines. Column (3) is the value of $\log [\mathrm{NII}] / \mathrm{H} \alpha$ using $\mathrm{H} \alpha$ corrected as above. Columns (4) and (5) are the uncorrected equivalent widths of the second two lines and column (6) is $\log [\mathrm{OIII}] / \mathrm{H} \beta$ using uncorrected $\mathrm{H} \beta$. Emission lines have negative values and absorption lines are positive. 


\section{DERIVED METAL ABUNDANCES AND AGES}

Below we use the combined indices defined as follows:

$$
\begin{gathered}
<\mathrm{Fe}>=(F e 5270+F e 5335) / 2 \\
{[\mathrm{MgFe}]^{\prime}=\sqrt{\left(\mathrm{Mg}_{\mathrm{b}} \times[0.72 \times \mathrm{Fe} 5270+0.28 \times \mathrm{Fe} 5335]\right)}}
\end{gathered}
$$

With our 26 galaxy sample and the four measured line indices, we did not carry out a simultaneous multidimensional fit to determine the ages and metal abundances (e.g. Nelan et al. 2005). As a first approximation, we estimated $[\alpha / \mathrm{Fe}]$ and $[\mathrm{Z} / \mathrm{H}]$ for the VGs using the grid of models by Thomas et al. (2003; hereafter TMB) assuming an age of $12 \times 10^{9}$ years for all objects. This gives a reasonable mean value for $[\alpha / \mathrm{Fe}]$ but not $[\mathrm{Z} / \mathrm{H}]$ which depends more strongly on age. As a second step, we subdivided the sample by age and redetermined the metal abundances using the mean $[\alpha / \mathrm{Fe}]$. These results are discussed in $\S 5$.

\subsection{Alpha and Metal Element Abundances}

Figure 7 shows the observed $\mathrm{Mg}_{b}$ and $<\mathrm{Fe}>$ indices with error bars from Table 2 plotted on the TMB grid of constant $[\mathrm{Z} / \mathrm{H}]$ and $[\alpha / \mathrm{Fe}]$ for $12 \times 10^{9}$ year models. Whilst the galaxies widely span $[\mathrm{Z} / \mathrm{H}]$, most lie within $[\alpha / \mathrm{Fe}]=0.0$ to 0.3 and lie near a mean of about 0.15. Compared with similar plots for cluster galaxies (e.g., Coma; Mehlert et al. 2003), Thomas et al. (2005), and Bernardi et al. (2006), higher [Z/H] values corresponding to the upper right portion of the figure are typical, but a tail of objects fall into the lower left region where eight, mostly emission line galaxies, have apparent $[\mathrm{Z} / \mathrm{H}]$ from about -0.33 to below -2.25 . Normal ellipticals do not occupy this region of the diagram, but such low values $[\mathrm{Z} / \mathrm{H}]$ are found for many globular clusters which also lie in this portion of Figure 7 (Trager et al.1998). However, although the globular clusters are known to be old, an alternative interpretation of the eight VGs is that they are young and have higher $[\mathrm{Z} / \mathrm{H}]$.

Initial estimates of $[\alpha / \mathrm{Fe}]$ were obtained following Figure 4 in TMB using $\left(\frac{M g_{b}}{\langle F e\rangle}\right)$ which they demonstrate is insensitive to age and metal content. This method yields a median of 0.15 and a mean of $\overline{[\alpha / F e]}=0.16 \pm 0.03$ for our VGs excluding the two most negative values. This result is consistent with cluster early-type galaxies (e.g., Nelan et al. 2005) and as shown in $\S 4.3$, is little affected by the choice of age.

Such age insensitivity does not hold for $[\mathrm{Z} / \mathrm{H}]$ because of how curves of constant $[\mathrm{Z} / \mathrm{H}]$ 
shift in the $<\mathrm{Fe}>-\mathrm{Mg}_{b}$ diagram. Qualitatively, a given galaxy placed in a $<\mathrm{Fe}>-\mathrm{Mg}_{b}$ diagram of fixed age, such as Figure 7 , has higher $[\mathrm{Z} / \mathrm{H}]$ using younger models. Also the young metal poor objects are most strongly affected. This can be seen from the following two examples: Object 22 has $[\mathrm{Z} / \mathrm{H}]=-2.3$ assuming an age of $12 \times 10^{9}$ years, but $[\mathrm{Z} / \mathrm{H}]$ $=-1.1$ if it is $1 \times 10^{9}$ years old. Object 2 , apparently older and having higher metals, has $[\mathrm{Z} / \mathrm{H}]=0.00$ if it is $12 \times 10^{9}$ years old, but using $15 \times 10^{9}$ years only changes $[\mathrm{Z} / \mathrm{H}]$ to -0.03 . Therefore objects that are of low age and $[\mathrm{Z} / \mathrm{H}]$ the most sensitive to changes in model age.

\subsection{Age Estimates}

\subsubsection{The Weak or No-emission Line Galaxies}

Ages, $\tau$, for most of the VGs could be obtained next using Figure 8 and data in Table 2. Fortunately age depends weakly on $[\mathrm{Z} / \mathrm{H}]$ if $[\alpha / \mathrm{Fe}]$ is known and the grid was interpolated from TMB employing $[\alpha / F e]=0.15$. Twenty of the galaxies are covered by the TMB models and yield consistent ages and metallicities although nine of these have some detectable emission in Table 3.

We stress that in practice using $\mathrm{H} \beta$ makes this problematical due to the emission, for in Figure 8 and the $[\mathrm{MgFe}]^{\prime}-\mathrm{H} \beta$ plot, $\mathrm{H} \beta$ is essentially the age indicator. Our data do not currently cover $\mathrm{H} \gamma$ which suffers less from emission ( See e.g., Schiavon 2007; Bernardi et al. 2006; Poggianti et al. 2001a for discussions.) and tends to deliver ages lower by $\sim 10^{9}$ years. Nevertheless, the $\mathrm{H} \beta$ corrections are small and the ages are relatively unaffected.

\subsubsection{The Strong Emission Line Galaxies}

Six of the galaxies $(1,12,21,22,25$, and 26) lie outside the grid in Figure 8 and require comment as their ages cannot be constrained using this diagram.

Objects (1 and 12) lie just below the 15 Gyr isochrone in Figure 8. Their spectra show the strong features typical of early-type galaxies including $\mathrm{Mg}_{b}, \mathrm{NaD}$, and Fe lines. They both require $\mathrm{H} \beta$ emission corrections and it is plausible that these corrections are too small and they belong with the other objects of similar $<\mathrm{Fe}>$ in Figure 8. We have assigned these galaxies values of $\log \tau=10.2 \pm 0.5$.

Objects (21 and 26), after correction for emission, still lie far below the 15 Gyr isochrone, which naively would indicate ages $\gg 15$ Gyr. Number 21 has the strongest $\mathrm{H} \beta$ emission of all our VGs and number 26 has the bluest $(B-R)$ color. Although one cannot definitely 
determine whether these objects are either metal poor, young, or both with the current data, their non-typical blue spectra suggest that they contain young stars. In addition to the TMB models, Schiavan's (2007) and Bruzual \& Charlot's (2003) Lick indices with varying metal content and age but with only solar $\alpha$ abundance demonstrate that age-line strength relations depend strongly on metal content for young objects. For $[\mathrm{MgFe}]^{\prime}$ and Fe5270, when $[\mathrm{MgFe}]^{\prime}$ $<2$, inferred age drops significantly for a wide abundance range and would indicate ages $\tau<3$ Gyr for these two galaxies.

Objects (22 and 25) also have strong emission lines and lie outside Figure 8, far above the other galaxies. These would also appear to have ages, well below 3 Gyr, but cannot be constrained better.

\subsection{Final Metal Abundance Estimates}

Given the ages, $\tau$ determined from Figure 8, we have subdivided the 22 galaxies by age and determined $[\alpha / F e]$ and $[\mathrm{Z} / \mathrm{H}]$ along with estimates of their errors using the $\mathrm{Mg}_{b}$ $<\mathrm{Fe}>$ diagrams from the TMB models of age $\tau_{T M B}$ :

1. $\tau \geq 9 \mathrm{Gyr}, \tau_{T M B}=12 \mathrm{Gyr}$

2. $9>\tau \geq 5$ Gyr, $\tau_{T M B}=7$ Gyr

3. $5>\tau \geq 3 \mathrm{Gyr}, \tau_{T M B}=4 \mathrm{Gyr}$

4. $3>\tau \geq 2 \mathrm{Gyr}, \tau_{T M B}=2 \mathrm{Gyr}$

5. $\tau<2$ Gyr, $\tau_{T M B}=1$ Gyr

The metal abundances have been eliminated for the four galaxies (21, 22, 25, and 26). These would yield $[\mathrm{Z} / \mathrm{H}]=-0.65$ to -1.74 assuming $\tau=1$ Gyr. As explained above, it is impossible to determine their ages using $\mathrm{H} \beta$ and $<\mathrm{Fe}>$ with the present data. It appears that diagrams such as Figure 7 cannot be employed if the stellar populations are younger than a few Gyr. Using Schiavon's (2007) Lick indices for single stellar populations for solarscaled and $\alpha$-enhanced isochrones that cover a more limited range of metallicities, one sees that the $\mathrm{Mg}_{b}$ or $<\mathrm{Fe}>$ indices for these four galaxies could be fit with models of age $\tau=$ 0.2 - $0.3 \mathrm{Gyr}$ and $[\mathrm{Z} / \mathrm{H}]$ values reduced much less and closer to solar.

Figure 9 gives histograms of the derived metal abundances. For $[\alpha / F e]$ the distribution is fairly narrow with an average $\overline{[\alpha / F e]}=0.15 \pm 0.09$ and a median of 0.13 . This confirms 
the first estimate. The distribution of $[\mathrm{Z} / \mathrm{H}]$ is somewhat broader and shows a tail towards lower values. The run of $[\mathrm{Z} / \mathrm{H}]$ is similar to our first approximation for the older galaxies, but the quite metal poor ones now have higher values of $[\mathrm{Z} / \mathrm{H}]$. The mean is $\overline{[Z / H]}=0.15 \pm 0.28$ and a median of 0.22 . These data are considered further in $\S 5$.

\subsection{Summary of the Age and Metal Abundance Estimates}

Table 4 presents the derived age and abundance parameters for the 26 VGs. Column (1) is the identification number from the other tables. Columns (2) and (3) are the logarithm of the age in Gyr, $\log \tau$ and its error from Figure 8 . The six galaxies located outside the range of the models in Figure 8 as discussed in $\S 4.2 .2$ are indicated with corresponding symbols. Columns (4) and (5) are $[\mathrm{Z} / \mathrm{H}]$ and its error using the galaxy's position in the $\mathrm{Mg}_{b}-<\mathrm{Fe}>$ diagrams listed above and columns (6) and (7) are the equivalent for $[\alpha / \mathrm{Fe}]$. The errors reflect the observational uncertainties, are the average of upper and lower values, and do not include uncertainties in the theoretical relations.

\section{DISCUSSION OF THE VG MEASUREMENTS}

\subsection{Comparison with Cluster Galaxies}

Figure 10 shows the histogram of VG ages derived using Table 4. Galaxies with detected $\mathrm{H} \beta$ emission are shaded. We place objects ( 1 and 12) in the $>9$ column of Figure 10. The two galaxies (21 and 26) are indicated in the $\gg 15$ column, but adopting Bruzual \& Charlot's (2003) relation would move them into the $\tau<3$ Gyr column of Figure 10 where they also appear as the wider hatched section. Sources (22 and 25) also appear in the $\tau<3$ column.

We compare the age distribution in Figure 10 with the $\mathrm{H} \beta$ ages of Coma early-type galaxies given in Fig. 7 of Poggianti et al. (2001a) for their most luminous, $R<15$ brightness subsample. Binning as Poggianti et al. (2001a) in four age bins, $\tau$ (viz. $\tau<3,3-5,5-9$, and $>9 \mathrm{Gyr}$ ), there are $7,4,8$, and $7 \mathrm{VGs}$ respectively. This is a higher proportion of young VG galaxies relative to the Coma sources where the corresponding numbers are 4, 4, 8, and 25 in their inner Coma 1 region where there is a higher proportion of objects in the $\tau>9$ Gy bin. Coma appears to show an increase in the younger bins for decreasing luminosity, but not to the extent of the VGs.

Rejecting the 10 galaxies with the largest $\mathrm{H} \beta$ emission corrections (as do some authors, e.g., Nelan et al. 2005; Smith et al. 2006), this leaves a subset of sources in the unshaded 
bins and the numbers of objects become $0,3,8$, and 5 in the same bins respectively, raising the ages, but still averaging younger than for the cluster objects.

Figure 11 compares age, metal abundances, and luminosities with $\log \sigma$. We reject the values of $[\mathrm{Z} / \mathrm{H}]$ for objects $(21,22,25$, and 26) for the reasons discussed above. The regression relations for $[\mathrm{Z} / \mathrm{H}],[\alpha / \mathrm{Fe}]$, and age for cluster early-types found in Nelan et al. (2005) and Fig. 14 of Bernardi et al. (2006) are shown, as is the Faber-Jackson relation, $\left(M_{B}, \log \sigma\right)$, from Dressler et al. (1987) for the Coma cluster. Nelan et al. (2005) further discuss the range of values in these relations in the literature. The Figure 11 plots show two features: Firstly, most of the higher $\sigma$ VGs lie near the mean lines for the cluster galaxies. Secondly, the $\log \sigma-M_{B}$ relation is similar to that of Coma and that of Kuntschner et al. (2002) for their void sample.

Finally, using the $\mathrm{H} \beta$ ages of the VGs, Figure 12 shows that most lie near the mean $[\alpha / \mathrm{Fe}]$ relations found by Mehlert et al. (2003) for Coma cluster early types and Fig. 13 of Bernardi et al. (2006) for ellipticals in different environments. However, in the $[\mathrm{Z} / \mathrm{H}]$ diagram some younger objects fall below the mean relations.

\subsection{Properties of the Emission line Objects}

\subsubsection{The diagnostic diagram for the VGs}

The presence of $\mathrm{H} \alpha, \mathrm{H} \beta$, [NII], and [OIII] emission in our sample indicates ongoing star formation. For our VGs, 12 of 26 or $46 \%$ are objects show detectable $\mathrm{H} \beta$ and [OIII] emission lines and 18 show at least one of $\mathrm{H} \alpha$ or $[\mathrm{NII}] \lambda 6583$. Nelan et al. (2005) found $\sim 12 \%$ of their early-type cluster galaxies had emission. Pimbblet et al. (2006) find $20 \%$ for their composite sample of X-ray bright galaxies in clusters at $z \sim 0.1$. Studies of the "E $+\mathrm{A}$ " galaxies (Zabludoff et al. ; 1996 and Quintero et al. ; 2004), although not showing emission lines are thought to be products of recent star formation, indicate that in general such early-type galaxies are rarer, currently $\sim 1 \%$ of the general field. The 8 VGs with no detected emission lines are more luminous than those with detected emission. The mean values of the two types are $\overline{M_{K}}=-23.9$ and $\overline{M_{K}}=-23.3$ respectively.

Figure 13 plots the data in Table 3 of $\log [\mathrm{NII}] / \mathrm{H} \alpha$ and $\log [\mathrm{OIII}] / \mathrm{H} \beta$ in the diagnostic diagram which separates H II star-forming regions from active galactic nuclei (Osterbrock 1989). The loci dividing the galaxy classes are from Kewley et al. (2001), Kauffmann et al. (2003), and Yan et al. (2006). The underlying Balmer absorption is required for some of these galaxies as can be seen from the spectra of galaxies 4 and 24 in Figure 4 where [NII] $\lambda 6583$ is stronger than $\mathrm{H} \alpha$; this appears to result from emission filling underlying absorption in 
the $\mathrm{H} \alpha$ line. The 2.32 Åaverage equivalent width of $\mathrm{H} \alpha$ in absorption was added to $\mathrm{H} \alpha$ as described above and moves some of the points left in Figure 13. It appears that of the 12 galaxies in Figure 13, six objects are star-forming, two are Seyferts, three are liners, and one borders the liners and star-forming regions.

For comparison, several investigators including Goudfrooi (1999) and Sarzi et al. (2006), discuss the $\mathrm{H} \alpha$ emission in giant ellipticals and conclude that the liner type are the most numerous. Smith, Lucey \& Hudson (2007) studied the occurrence of emission among lowluminosity galaxies in the Shapley supercluster and find that $\sim 20 \%$ of their early-type redsequence members show emission features. Although this is a lower percentage than for the VGs, their diagnostic diagram seems to show a similar mix of types. They report that their most luminous galaxies brighter than about $M_{R}=-21.5 \mathrm{mag}$. are mostly AGN/LINERs, while galaxies fainter than this are mostly star forming. Our small sample appears to agree with this. The two Seyferts in Figure 13 are the sample's most luminous with $\overline{M_{R}}=-21.5$ mag., while the Seyferts plus liners have $\overline{M_{R}}=-20.2 \mathrm{mag}$. and the star forming galaxies give an average of $\overline{M_{R}}=-19.2 \mathrm{mag}$.

\subsubsection{Star formation rates}

To estimate star formation rates, we take

$$
\operatorname{SFR}\left(M_{\odot} y r^{-1}\right)=\frac{L(H \alpha)}{1.26 \times 10^{41} \mathrm{ergssec}^{-1}}
$$

(Kennicut 1998). Using flux calibrated $\mathrm{H} \alpha$ and $\mathrm{H} \beta$ data described above, it is possible to estimate SFR for six galaxies that have strongest $\mathrm{H} \alpha$ and $\mathrm{H} \beta$ emission (numbers 15, 17, 21, 22,23 , and 25) and for which we have photometric calibrations. We assumed the intrinsic unreddened $\mathrm{H} \alpha / \mathrm{H} \beta$ ratio is 2.85 and the average extinction curve to derive the extinction parameter, $c$ (Osterbrock 1989), which has values $c=0.2-0.8$. In $\S 3.2 .2$ we took the mean observed $\mathrm{H} \alpha / \mathrm{H} \beta$ ratio for the whole sample to be 4.5 for the emission correction to $\mathrm{H} \beta$; this

gives $c=0.5$ which is close to the mean of these six objects. The observed $\mathrm{H} \alpha$ emission fluxes range from $2 \times 10^{-14}$ to $4 \times 10^{-14} \mathrm{ergssec}^{-1} \mathrm{~cm}^{-2}$. and thus indicate $S F R \sim 0.2$ to $1.0 \mathrm{M}_{\odot} \mathrm{yr}^{-1}$, moderate values (Kewley Geller \& Jansen 2004; Salzer et al. 2005).

Star formation rates of this order of magnitude are also observed at a lower frequency in many early-type galaxies. Yi et al. (2005) used the all sky GALEX near ultraviolet colormagnitude diagram to study star formation in nearby bright early-type galaxies and conclude that $\sim 15 \%$ of these objects show low-level star formation. 


\section{CONCLUSIONS}

Spectroscopic data are given for 26 early-type void galaxies that were listed in Grogin \& Geller $(1999 ; 2000)$. The morphologies of our VGs are all E/S0. Most of them lie in the red sequence for elliptical galaxies, belong to the brighter end of the luminosity function appropriate for void galaxies, and are not intrinsically faint void objects for which environmental differences are expected to be more pronounced.

Based on the spectroscopy, our main observational results are:

(1) The values of $[\alpha / \mathrm{H}]$ lie near 0.15 which is close to that of cluster ellipticals.

(2) The metal abundances, $[\mathrm{Z} / \mathrm{H}]$, found from $\mathrm{Mg}_{b}$ and $\left.<\mathrm{Fe}\right\rangle$, average close to 0.15 , normal for early-types in clusters, but some VGs seem to have lower metal content than found in clusters but this interpretation is clouded by the higher sensitivity of $[\mathrm{Z} / \mathrm{H}]$ for the low ages..

(3) Compared to the early-type cluster galaxies studied, e.g., in Nelan et al. (2005), the runs of $[\alpha / \mathrm{H}]$ and $[\mathrm{Z} / \mathrm{H}]$ with $\sigma$ and age for the VGs seem to be consistent in many cases, but there are some objects that appear to have lower ages and metals, $[\mathrm{Z} / \mathrm{H}]$.

(4) Ages for the stellar populations in the VGs were determined from the $(<\mathrm{Fe}>$, $\mathrm{H} \beta$ ) diagram and required corrections to $\mathrm{H} \beta$ for emission that were derived from $\mathrm{H} \alpha$. The resulting ages of several of the VGs are younger than those found by other investigators for bright early-types in denser environments such as Coma. This is particularly the case for VGs with emission lines that comprise the majority of galaxies younger then 5 Gyr. There is a reduced percentage of old objects $(\tau>9$ Gyr in the distribution which indicates a range of old and young galaxies.

(5) The occurrence of emission in the VG spectra is high and appears to agree with the age conclusions. In the sample, $46 \%$ show detectable $\mathrm{H} \beta$ lines and $69 \%$ have $\mathrm{H} \alpha$, different from early-types in higher density environments.

(6) The positions of 12 VGs in the diagnostic diagram indicate a mixture of emission types, but the signature of star formation is common with moderate values of star formation in the range $S F R \sim 0.2$ to $1.0 \mathrm{M}_{\odot} / \mathrm{yr}$.

(7) Visual inspection of blue POSS-II images find that five or $19 \%$ present evidence of shell or ring structures.

The early-type VGs of this study appear to differ in age from their cluster counterparts. Some of these trends have been indicated by other investigations referenced in the introduction, but here we found evidence for a prevalence of strong emission lines and younger ages. 
The most massive early-type VGs, judged from their luminosities and velocity dispersions, tend to have the highest ages and $\alpha$-enrichments as do their cluster counterparts.

The results for metal abundances and age determinations depend on the applicability of the TMB models that have been employed to obtain them. In addition the ages of 10 VGs depend critically on the interpretation of the $\mathrm{H} \beta$ line strengths. Although we have conducted a straightforward interpretation of the data using these models, the interplay of age, $\alpha$, and $\mathrm{Z}$ make determination of these quantities tricky at best for the youngest objects which also are the most interesting; they are the most unreliable. New generations of models which cover a wider range of variation in element abundances and which handle multiple starbursts should aid in this (e.g., Schiavon 2007).

Nevertheless, there are morphological differences between the VGs and the cluster earlytypes that are model independent. These include the incidence of more emission lines, shells, and rings, suggesting that many VGs are still undergoing hierarchical assembly. These results are unlikely to go away. In the context of current thinking on galaxy formation, these findings for the early-type VGs seem consistent with the "downsizing" picture (e.g., De Lucia et al.2006; Nelan et al.2005; Smith et al.2006) in which the star formation and evolution stop earlier for the more massive objects and are drawn out longer in time for the lower mass ones. The younger ages would be consistent with a slower evolution in the lower density environments.

Studies of void evolution seem to be consistent with these results, but indicate that a simple process of galaxy formation is unlikely. Void structures would form differently from those in the higher density regions. Many authors including, e.g., Sheth \& van de Weygaert (2004), Colberg et al. (2005), and van de Weygaert \& van Kampen (1993) indicate that the large voids arose from the mergers of smaller voids which get squeezed down as overdensities in the large voids as they collapse. Radial galaxy movements are suppressed and their peculiar motions are mostly confined to run along the void walls where most of the galaxies reside. This leads to VGs in large voids forming in the wall regions of the smaller voids as they collapse and remain in the voids. Thus the observed VGs would arise from a 'cloud-in-void' process (Sheth \& van de Weygaert 2004) which naturally produces a lower density environment.

The problem of constructing an uncontaminated VG sample which detects the fainter void interior structures remains. More detailed mapping of the VGs kinematical and line strength structures as well as investigation of their immediate environments would help resolve some of these questions.

We wish to thank Drs. R. P. Saglia and J. R. Lucey who made comments on earlier ver- 
sions of this paper and the referee, Dr. R. P. Schiavon for careful reading of the manuscript and suggestions that improved the paper. This research has made use of the NASA/IPAC Extragalactic Database (NED) which is operated by the Jet Propulsion Laboratory, California Institute of Technology, under contract with the National Aeronautics and Space Administration. The Digitized Sky Surveys were produced at the Space Telescope Science Institute under U.S. Government grant NAG W-2166. The images of these surveys are based on photographic data obtained using the Oschin Schmidt Telescope on Palomar Mountain and the UK Schmidt Telescope. The plates were processed into the present compressed digital form with the permission of these institutions. The Second Palomar Observatory Sky Survey (POSS-II) was made by the California Institute of Technology with funds from the National Science Foundation, the National Geographic Society, the Sloan Foundation, the Samuel Oschin Foundation, and the Eastman Kodak Corporation. The Oschin Schmidt Telescope is operated by the California Institute of Technology and Palomar Observatory.

\section{REFERENCES}

Benson, A. J., Frenk, C. S., Baugh, C. M., Cole, S., \& Lacey, C. G. 2001, MNRAS, 327, 1041

Bernardi, M., Renzini, A., da Costa, L. N., Wegner, G., Alonso, M. V., Pelegrini, P. S., Rite, C., Maia, M. A. G., \& Wilmer, C. N. A. 1998, ApJ, 509, L143

Bernardi, M. et al. 2003a, AJ, 125, 1866

Bernardi, M. et al. 2003b, AJ, 125, 1882

Bernardi, M., Nichol, R. C., Sheth, R. K., Miller, C. J., \& Brinkmann, J. 2006, AJ, 131, 1288

Bruzual, G. \& Charlot, S. 1993, ApJ, 405, 538

Bruzual, G. \& Charlot, S. 2003, MNRAS, 344, 1000

Carter, D., Mobasher, B., Bridges, T. J., Poggianti, B. M., Komiyama, Y., Kashikawa, N., Doi, M., Iye, M., Sekiguchi, M., Shimasaku, K., Yagi, M., \& Yasuda, N. 2002, ApJ 567,772

Colbert, J. W., Mulchaey, J. S., \& Zabludoff, A. I. 2001, AJ, 121, 808

Cole, S., Lacey, C. G., Baugh, C. M., \& Frenk, C. S. 2000, MNRAS, 319, 168 
Colless, M., Burstein, D., Davies. R. L., McMahan, R. K., Saglia, R. P., \& Wegner, G. 1999, MNRAS, 303813

Colberg, Sheth, R. K., Diaferio, A., Gao, L., \& Yoshida, N. 2005, MNRAS, 360, 216

Colobert, M., Sarzi, M., Daview, R. L., Kuntschner, H., \& Colless, M. 2006, MNRAS

Croton, D. J. et al. 2005, MNRAS, 356, 1155

Croton, D. J. et al. 2006, MNRAS, 365, 11

De Lucia, G., Springel, V., White, S. D. M.,Croton, D., \& Kauffmann, G. 2006, MNRAS, 366,499

Dressler, A., Lynden-Bell, D., Burstein, D., Davies, R. L., Faber, S. M.,Terlevich, R. J., \& Wegner, G. 1987, ApJ, 313, 42

de Vaucouleurs, G., de Vaucouleurs, A., Corwin, H. G. Jr., Buta, R. J., Paturel, G., \& Foque, P. 1991, Third Reference Catalogue of Bright Galaxies (New York: Springer)

Edwards, S. A., Colless, M., Bridges, T. J., Carter, D., Mobasher, B., \& Poggianti, B. 2002, ApJ, 567, 178

Efstathiou, G. et al. 2002, MNRAS, 320, 29

Faber, S. M. et al. 2005, astro-ph/0506044

Falco, E. E., Kurtz, M. J., Geller, M. J., Huchra, J. P., Peters, J., Berlind, P., Mink, D. J., Tokarz, S. P., \& Elwell, B. 1999, PASP, 111, 438

Geller, M. J., \& Huchra, J. P. 1989, Science, 246, 897

Geller, M. J., Kurtz, M. J., Wegner, G., Thorstensen, J. R., Fabricant, D. G., Marzke, R. O., Huchra, J. P., Schild, R. E., Falco, E. E. 1997, AJ, 114, 2205

Geller, M. J., et al. in preparation

Glazebrook. K. et al. 2004, Nature, 430, 181

Gonzales, J. J. 1993, Ph.D.Thesis, Univ. Calif. Santa Cruz

Goudfrooi, P. 1999, in Star Formation in Early-type Galaxies, ASP Conference Series, Vol. 163, J. Cepa \& P. Carral, eds., 55

Grogin, N. A. \& Geller, M. J. 1999, AJ, 118, 2561 (GG99) 
Grogin, N. A. \& Geller, M. J. 2000, AJ, 119, 32 (GG00)

Hopkins, P. F. et al. 2006, ApJS, 163, 1

Hoyle, F., Rojas, R. R., Vogley, M. S., \& Brinkmann, J. 2005, ApJ, 620, 618

Jerjen, H. \& Tammann, G. A. 1997, A\&A, 321, 713

Jørgensen, I., Franx, M., \& Kjaergaard, P. 1995, MNRAS, 276, 1341

Kauffmann, G,. et al. 2003, MNRAS, 346, 1055

Kauffmann, G., et al. 2006, MNRAS, 353, 713

Kennicut, R. C, Jr. 1998, ApJ, 498, 541

Kewley, L. J. et al. 2001, ApJ, 556, 121

Kewley, L. J., Geller, M. J. \& Jansen, R. A. 2004, AJ, 127, 2002

Kochfar, S. Silk, J. 2006, MNRAS, 370, 902

Kuntschner, H., Lucey, J. R., Smith, R. J., Hudson, M. J., \& Davies, R. L. 2001, MNRAS, 323,615

Kuntschner, H., Smith, R. J., Colless, M., Davies, R. L., Kaldare, R., Vazdekis, A. 2002, MNRAS, 337, 172

Mehlert, D., Thomas, D., Saglia, R. P., Bender, R., Wegner, G. 2003, A\&A, 407, 423

Mobasher, B. et al. 2001, ApJS, 137, 279

Nelan, J. E., Smith, R. J., Hudson, M. J., Wegner, G. A., Lucey, J. R., Moore, S. A. W., Quinney, S. J., \& Suntzeff, N. B. 2005, ApJ, 632, 137

Nusser, A., Gubser, S. S., \& Peebles, P. J. E. 2005, Phys. Rev. D, 71, 083505

Ogando, R. L. C., Maia, M. A. G., Chiappini, C., Pellegrini, P. S., Schiavon, R. P., \& da Costa, L. M. 2005, ApJ, 632, L61

Osterbrock, D. E. 1989, Astrophysics of Gaseous Nebulae and Active Galactic Nuclei (Mill Valley: University Science Books)

Patiri, S. G. et al. 2006, MNRAS, 372, 1710

Peebles, P. J. E. 2001, ApJ. 557, 495 
Pimbblet, K. A., Smail, I., Edge, A. C., O'Hely, E., Couch, W. J., \& Zabludoff, A. I. 2006, MNRAS, 366, 645

Poggianti, B., Bridges, T. J., Mobasher, B., Carter, D., Doi, M., Iye, M., Kashikawa, N., Komiyama, Y., Sekiguchi, M., Shimasaku, K., Yagi, M., \& Yasuda, N. 2001a, ApJ 562,689

Poggianti, B., Bridges, T. J., Carter, D., Mobasher, B., Doi, M., Iye, M., Kashikawa, N., Komiyama, Y., Okamura, S., Sekiguchi, M., Shimasaku, K., Yagi, M., \& Yasuda, N. 2001b, ApJ, 563118

Quintero, A. D. et al. 2004, ApJ, 602, 190

Rines, K., Geller, M. J., Kurtz, M. J., \& Diaferio, A. 2005, AJ, 130, 1482

Rojas, R. R., Vogley, M., Hoyle, F., \& Brinkman, J. 2005, ApJ, 624, 571

Salzer, J. J. et al. 2005, ApJ, 624, 661

Sarzi, M. et al. 2006, MNRAS, 366, 1151

Schlegel, D. J., Finkbeiner, D., \& Davis, M. 1998, ApJ, 500, 525

Schiavon, R. P. et al. 2006, ApJ, 651, 93L

Schiavon, R. P. 2007, ApJS, 171, 146

Sheth, R. K. \& van de Weygaert, R. 2004, MNRAS, 350, 517

Sil'chenko, O. K. 2006, A\&A, 641, 229

Skrutskie, M. F., et al. 2006, AJ, 131, 1163

Smith, R. J. et al. 2004, AJ, 128, 1558

Smith, R. J., Hudson, M. J., Lucey, J. R., Nelan, J. E., \& Wegner, G. A. 2006, MNRAS, 369,1419

Smith, R. J., Lucey, J. R., \& Hudson, M. J. 2007, astro-ph/0707.1695

Spergel, D. N. et al. 2003, ApJS, 148, 175

Stasińska, G., Mateus Jr., A., Sodre Jr., L., \& Szczerba, R. 2004, A\&A, 420, 475

Tantalo, R. \& Chiosi, C. 2002, A\&A, 388, 396 
Thomas, D., Maraston, C., \& Bender, R. 2003, MNRAS, 339, 897 (TMB)

Thomas, D., Maraston, C., Bender, R., \& Mendes de Oliveira, C. 2005, ApJ, 621, 673

Tinsley, B. M. 1978, ApJ, 222, 14

Tonry, J. \& Davis, M. 1979, AJ, 84, 1511

Trager, S. C., Worthey, G., Faber, S. M., Burstein, D., Gonzales, J. J. 1998, ApJS, 116,1

Wegner, G., Colless, M., Saglia, R. P., McMahan, Davies, R. L., Burstein, D., \& Baggley, G. 1999, MNRAS, 305, 259

Wegner, G., et al. 2001, AJ, 122, 2893

Wegner, G., Corsini, E. M., Saglia, R. P., Bender, R., Merkl, D., Thomas, D., Thomas, J., \& Mehlert, D. 2002, A\&A, 395, 573

Wegner, G., Bernardi, M., Willmer, C. N. A., da Costa, L. N., Alonso, M. V., Pellegrini, P. S., Maia, M. A. G., Chaves, Rite, C. 2003, AJ, 126, 2268

Worthey, G. 1994, ApJS, 95, 105

Worthey, G., Faber, S. M., Gonzalez, J., \& Burstein, D. 1994, ApJS, 94, 687

Yan, R. et al. 2006, ApJ, 648, 281

Yi, S.-J. et al., 2005, ApJ, 619, L111

Zabludoff, A. I. et al. . 1996, ApJ, 466, 104 
Table 1. Basic Data for the Observed Void Galaxies

\begin{tabular}{|c|c|c|c|c|c|c|c|c|}
\hline (1) & $\begin{array}{c}\text { Name } \\
(2)\end{array}$ & $\begin{array}{c}\alpha \\
\mathrm{J} 2000 \\
(3)\end{array}$ & $\begin{array}{c}\delta \\
\mathrm{J} 2000 \\
(4)\end{array}$ & $\begin{array}{c}c z \\
\mathrm{~km} \mathrm{~s}^{-1}\end{array}$ & Type & $\begin{array}{c}M_{B} \\
\text { mag. } \\
(7)\end{array}$ & $\begin{array}{c}M_{R} \\
\mathrm{mag}, \\
(8)\end{array}$ & $\begin{array}{c}M_{K} \\
\text { mag. } \\
(9)\end{array}$ \\
\hline 1 & 15R 464.015505 & 000336.098 & +103612.54 & 8085 & S0 & -19.00 & -20.50 & -23.84 \\
\hline 2 & CGCG 0027.9+0536 & 003028.637 & +055139.06 & 7238 & $\mathrm{E} 2$ & -19.15 & -20.59 & -24.03 \\
\hline 3 & NGC 476 & 012019.952 & +160112.40 & 6439 & So & -18.78 & -20.37 & -23.81 \\
\hline 4 & IC 1700 & 012524.652 & +145152.43 & 6484 & E0(shells) & -20.12 & -21.64 & -25.01 \\
\hline 5 & CGCG 0133.6+1734 & 013620.281 & +174858.01 & 6065 & E2 & -18.10 & -19.71 & -23.11 \\
\hline 6 & CGCG $1348.4+3517$ & 135038.973 & +350218.09 & 6407 & E0 & -19.55 & -21.01 & -24.42 \\
\hline 7 & CGCG $1429.5+3929$ & 143127.815 & +391532.14 & 7038 & SB0 & -19.22 & -20.73 & -24.15 \\
\hline 8 & CGCG $1433.0+2311$ & 143517.143 & +225746.34 & 6336 & SB0(ring) & -18.54 & -19.84 & -23.06 \\
\hline 9 & CGCG $1434.7+4010$ & 143642.686 & +395637.64 & 6217 & $\mathrm{E} 1$ & -18.72 & -20.19 & -23.74 \\
\hline 10 & CGCG $1506.2+2208$ & 150829.040 & +215651.73 & 6259 & S0 & -18.82 & -20.42 & -23.72 \\
\hline 11 & 15R 328.038850 & 153341.477 & +280844.24 & 5772 & So/a & -16.95 & -18.35 & -21.73 \\
\hline 12 & CGCG $1536.8+3707$ & 153838.464 & +365730.18 & 5751 & S0 & -19.02 & -20.37 & -23.76 \\
\hline 13 & CGCG $1549.1+2551$ & 155113.249 & +254205.11 & 6546 & So & -19.33 & -20.85 & -24.18 \\
\hline 14 & CGCG $1552.1+3018$ & 155411.455 & +300921.35 & 6175 & E0 & -18.30 & -19.82 & -23.39 \\
\hline 15 & CGCG $1600.6+4302$ & 160216.663 & +425459.66 & 7541 & S0(ring) & -19.77 & -20.98 & -24.21 \\
\hline 16 & CGCG $1607.5+4152$ & 160916.555 & +414443.47 & 7891 & S0 & -19.37 & -20.81 & -24.15 \\
\hline 17 & CGCG 2108.7+0318 & 211115.074 & +0331 19.42 & 5342 & E3 & -18.17 & -19.51 & -22.79 \\
\hline 18 & NGC 7113 & 214226.650 & +123407.35 & 5989 & So & -19.20 & -20.89 & -24.59 \\
\hline 19 & CGCG $2140.0+1340$ & 214226.896 & +135358.61 & 5631 & E2 & -18.58 & -20.22 & -23.63 \\
\hline 20 & 15R 518.119354 & 215027.756 & +123808.91 & 6850 & So/a & -17.27 & -18.82 & -22.29 \\
\hline 21 & 15R 520.029201 & 223505.336 & +105525.89 & 5240 & E0 & -16.76 & -18.05 & -21.50 \\
\hline 22 & CGCG 2236.9+1336 & 223921.911 & +135254.19 & 5423 & S0(ring) & -17.93 & -18.66 & -20.89 \\
\hline 23 & NGC 7509 & 231221.430 & +143633.41 & 5118 & $\mathrm{E} 0+$ star & -19.36 & -21.01 & -24.37 \\
\hline 24 & NGC 7550 & 231516.007 & +185742.49 & 5340 & E0 & -20.29 & -21.98 & -25.45 \\
\hline 25 & 15R 523.044360 & 233738.094 & +120600.42 & 8104 & S0(shells) & -18.00 & -18.92 & -21.74 \\
\hline 26 & 15R 523.036286 & 234429.634 & +114048.01 & 7090 & SB0 & -18.98 & -20.38 & -23.09 \\
\hline
\end{tabular}


Table 2. The Spectroscopic Data for the Void Galaxies

\begin{tabular}{|c|c|c|c|c|c|c|c|c|c|c|c|c|c|c|}
\hline 1 & 1 & 7859 & 14 & 126 & 5 & 0.736 & 0.178 & 0.319 & 4.442 & 0.174 & 3.104 & 0.120 & 2.819 & 0.078 \\
\hline 2 & 2 & 7059 & 14 & 130 & 13 & 1.241 & 0.143 & 0.303 & 3.958 & 0.193 & 3.022 & 0.080 & 2.428 & 0.013 \\
\hline 3 & 2 & 6325 & 10 & 134 & 2 & 1.832 & 0.191 & -0.001 & 3.601 & 0.022 & 2.846 & 0.058 & 2.487 & 0.018 \\
\hline 6 & 1 & 6363 & 20 & 147 & 10 & 1.700 & 0.246 & -0.094 & 4.455 & 0.221 & 3.329 & 0.151 & 2.882 & 0.096 \\
\hline 7 & 1 & 6910 & 26 & 206 & 15 & 1.307 & 0.304 & 0.172 & 5.157 & 0.258 & 3.250 & 0.175 & 3.367 & 0.110 \\
\hline 8 & 1 & 6267 & 20 & 117 & 10 & 1.264 & 0.248 & 0.737 & 2.831 & 0.222 & 2.398 & 0.152 & 1.572 & 0.096 \\
\hline 9 & 1 & 6027 & 26 & 130 & 14 & 0.505 & 0.298 & 1.724 & 3.641 & 0.255 & 2.859 & 0.173 & 2.851 & 0.109 \\
\hline 10 & 1 & 6202 & 25 & 178 & 14 & 1.082 & 0.296 & 0.540 & 4.709 & 0.253 & 3.214 & 0.172 & 3.141 & 0.108 \\
\hline 11 & 1 & 5565 & 47 & 49 & 34 & -2.125 & 0.487 & 4.823 & 2.069 & 0.367 & 0.989 & 0.244 & 1.703 & 0.149 \\
\hline 16 & 2 & 7695 & 02 & 169 & 6 & 1.585 & 0.258 & 0.184 & 4.335 & 0.193 & 3.180 & 0.050 & 2.840 & 0.132 \\
\hline 17 & 2 & 5158 & 10 & 94 & 11 & -0.459 & 0.313 & 3.184 & 2.351 & 0.014 & 2.109 & 0.114 & 1.937 & 0.054 \\
\hline 18 & 2 & 5778 & 02 & 133 & 8 & 1.868 & 0.239 & 0.086 & 4.564 & 0.019 & 2.897 & 0.043 & 2.326 & 0.172 \\
\hline 19 & 2 & 5407 & 05 & 142 & 19 & 1.592 & 0.226 & 0.158 & 4.152 & 0.020 & 2.862 & 0.005 & 2.629 & 0.075 \\
\hline 20 & 1 & 6548 & 26 & 135 & 15 & 1.804 & 0.305 & 0.280 & 3.362 & 0.260 & 1.737 & 0.176 & 2.705 & 0.110 \\
\hline 21 & 2 & 5067 & 08 & - & - & -9.021 & 0.352 & 3.219 & 1.652 & 0.012 & 1.102 & 0.288 & 1.178 & 0.028 \\
\hline 22 & 1 & 5253 & 93 & 144 & 28 & -7.123 & 0.857 & 18.971 & 1.062 & 0.557 & 0.423 & 0.364 & 0.662 & 0.215 \\
\hline 23 & 2 & 4880 & 02 & 166 & 42 & 0.021 & 0.307 & 1.452 & 4.944 & 0.077 & 2.890 & 0.045 & 2.604 & 0.102 \\
\hline 24 & 2 & 5076 & 06 & 222 & 10 & 1.064 & 0.249 & 0.634 & 4.437 & 0.292 & 3.106 & 0.049 & 3.913 & 0.105 \\
\hline 25 & 1 & 7911 & 101 & - & - & -1.924 & 0.921 & 10.570 & 0.311 & 0.587 & 1.182 & 0.382 & -0.131 & 0.225 \\
\hline 26 & 1 & 6922 & 24 & 95 & 9 & 0.538 & 0.287 & 0.721 & 0.435 & 0.248 & 1.971 & 0.168 & 0.939 & 0.106 \\
\hline
\end{tabular}


Table 3. The Emission Line Data for the Void Galaxies

\begin{tabular}{|c|c|c|c|c|c|c|}
\hline ID & $\begin{array}{c}\mathrm{H} \alpha \\
\AA\end{array}$ & $\begin{array}{c}{[\mathrm{NII}] \lambda 6583} \\
\AA\end{array}$ & $\log [\mathrm{NII}] / \mathrm{H} \alpha$ & $\begin{array}{c}\mathrm{H} \beta \\
\AA\end{array}$ & $\begin{array}{c}{[\mathrm{OIII}] \lambda 5007} \\
\AA\end{array}$ & $\log [\mathrm{OIII}] / \mathrm{H} \beta$ \\
\hline (1) & $(2)$ & (3) & $(4)$ & $(5)$ & (6) & $(7)$ \\
\hline 01 & +0.886 & -0.958 & -0.175 & -0.287 & -0.671 & +0.369 \\
\hline 02 & +0.956 & -0.946 & - & +1.448 & - & - \\
\hline 03 & +2.361 & - & - & +2.069 & - & - \\
\hline 04 & -0.245 & -2.245 & - & +3.123 & - & - \\
\hline 05 & +2.241 & - & - & +2.356 & - & - \\
\hline 06 & +2.745 & - & - & +3.162 & - & - \\
\hline 07 & +1.545 & - & - & +2.145 & - & - \\
\hline 08 & -0.996 & -2.483 & -0.125 & -0.381 & -0.605 & +0.201 \\
\hline 09 & -5.346 & -6.021 & -0.105 & -0.987 & -2.246 & +0.391 \\
\hline 10 & -0.112 & -1.494 & - & +2.545 & - & - \\
\hline 11 & -19.385 & -6.500 & -0.524 & -6.483 & -4.554 & -0.153 \\
\hline 12 & -12.312 & -9.850 & -0.172 & -4.168 & -2.295 & -0.259 \\
\hline 13 & -0.151 & -0.979 & - & +2.073 & - & - \\
\hline 14 & +1.383 & - & - & +3.073 & - & - \\
\hline 15 & -16.410 & -8.310 & -0.353 & -2.985 & -1.522 & -0.292 \\
\hline 16 & +1.493 & - & - & +2.098 & - & - \\
\hline 17 & -12.008 & -8.245 & -0.240 & -2.194 & -3.753 & +0.233 \\
\hline 18 & +1.931 & - & - & +2.881 & - & - \\
\hline 19 & +1.608 & - & - & +2.112 & - & - \\
\hline 20 & +1.058 & -0.614 & - & +1.636 & - & - \\
\hline 21 & -12.164 & -19.570 & +0.131 & -14.502 & -12.656 & -0.059 \\
\hline 22 & -83.050 & -10.885 & -0.894 & -19.615 & -18.398 & -0.028 \\
\hline 23 & -4.215 & -7.365 & +0.052 & -0.717 & -3.645 & +0.706 \\
\hline 24 & -0.533 & -2.545 & -0.050 & -0.240 & -0.962 & +0.602 \\
\hline 25 & -45.25 & -11.265 & -0.625 & -3.976 & -4.050 & +0.008 \\
\hline 26 & -0.924 & -1.328 & - & +0.995 & - & - \\
\hline
\end{tabular}


Table 4. Age and Abundance Results for the Observed Void Galaxies

\begin{tabular}{clccccc}
\hline \hline ID & $\log \tau$ & $\epsilon_{\tau}$ & {$[\mathrm{Z} / \mathrm{H}]$} & $\epsilon_{[Z / H]}$ & {$[\alpha / F e]$} & $\epsilon_{[\alpha / F e]}$ \\
$(1)$ & $(2)$ & $(3)$ & $(4)$ & $(5)$ & $(6)$ & $(7)$ \\
& & & & & & \\
\hline 01 & 10.2 & 0.5 & 0.19 & 0.09 & 0.14 & 0.08 \\
02 & 10.1 & 0.2 & 0.01 & 0.06 & 0.11 & 0.07 \\
03 & 9.8 & 0.3 & 0.04 & 0.04 & 0.07 & 0.04 \\
04 & 9.6 & 0.2 & 0.31 & 0.08 & 0.09 & 0.08 \\
05 & 9.7 & 0.2 & 0.17 & 0.05 & 0.06 & 0.08 \\
06 & 9.9 & 0.3 & 0.24 & 0.09 & 0.10 & 0.09 \\
07 & 10.0 & 0.3 & 0.44 & 0.09 & 0.19 & 0.10 \\
08 & 9.9 & 0.3 & -0.27 & 0.07 & 0.23 & 0.14 \\
09 & 9.3 & 0.3 & 0.39 & 0.18 & 0.12 & 0.13 \\
10 & 9.9 & 0.3 & 0.44 & 0.10 & 0.18 & 0.10 \\
11 & 9.6 & 0.3 & -0.65 & 0.27 & 0.24 & 0.34 \\
12 & 10.2 & 0.5 & -0.12 & 0.04 & 0.08 & 0.06 \\
13 & 9.6 & 0.3 & 0.45 & 0.07 & 0.13 & 0.09 \\
14 & 10.0 & 0.3 & 0.22 & 0.04 & 0.36 & 0.06 \\
15 & 9.0 & 0.5 & 0.26 & 0.04 & 0.12 & 0.05 \\
16 & 9.8 & 0.2 & 0.33 & 0.08 & 0.13 & 0.09 \\
17 & 9.3 & 0.2 & -0.15 & 0.05 & 0.09 & 0.08 \\
18 & 9.7 & 0.3 & 0.24 & 0.08 & 0.30 & 0.06 \\
19 & 9.9 & 0.3 & 0.18 & 0.03 & 0.17 & 0.04 \\
20 & 9.8 & 0.3 & -0.12 & 0.09 & 0.18 & 0.14 \\
21 & $\gg 15$ & - & - & - & - & - \\
22 & $3<$ & - & -1 & - & - & - \\
23 & 10.2 & 0.3 & 0.22 & 0.04 & 0.30 & 0.06 \\
24 & 9.6 & 0.3 & 0.53 & 0.07 & 0.04 & 0.07 \\
25 & $3<$ & - & - & - & - & - \\
26 & $\gg 15$ & - & - & - & - & - \\
\hline & & & & & &
\end{tabular}



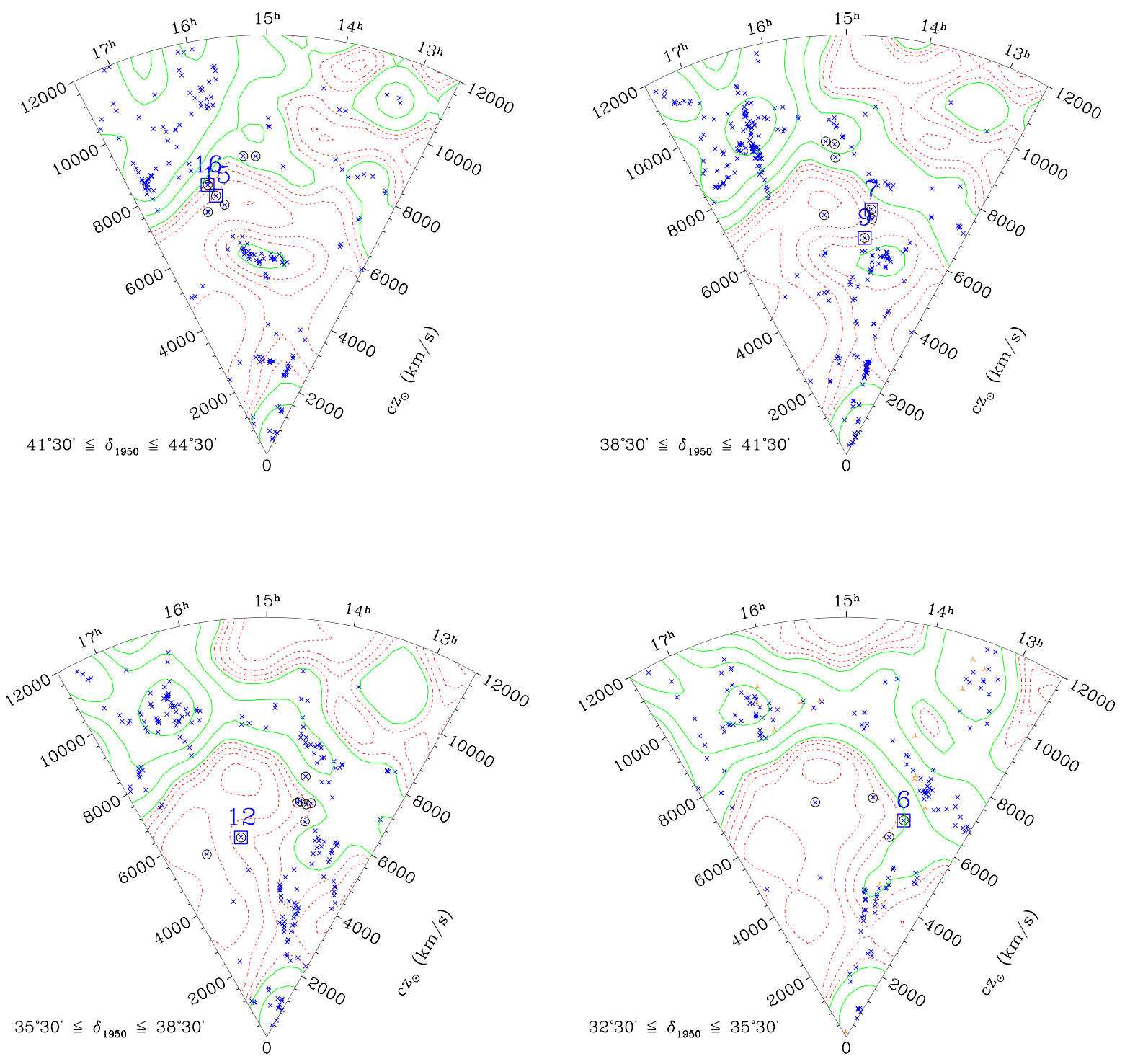

Fig. 1. - Eight successive $3^{\circ}$ declination slices delineating the northern void in this study. The CfA2 redshift survey galaxies are plotted with crosses, fainter 15R redshift survey galaxies with orange triangular crosses. The GG99 sample is circled. The subset of GG99 investigated in this study is marked by larger squares with labels above, referring to the reference number in Table 1. Blue and orange numbers are from CfA2 and 15R respectively. We overplot $5 h^{-1}$ Mpc-smoothed number density contours as determined from CfA2. Underdensities in $0.2 \bar{n}$ decrements are marked with dotted contours; overdensities in logarithmic intervals of $\bar{n}, 2 \bar{n}, 4 \bar{n}$, etc., are marked with solid contours. 
Fig. 1. - Cont'd.
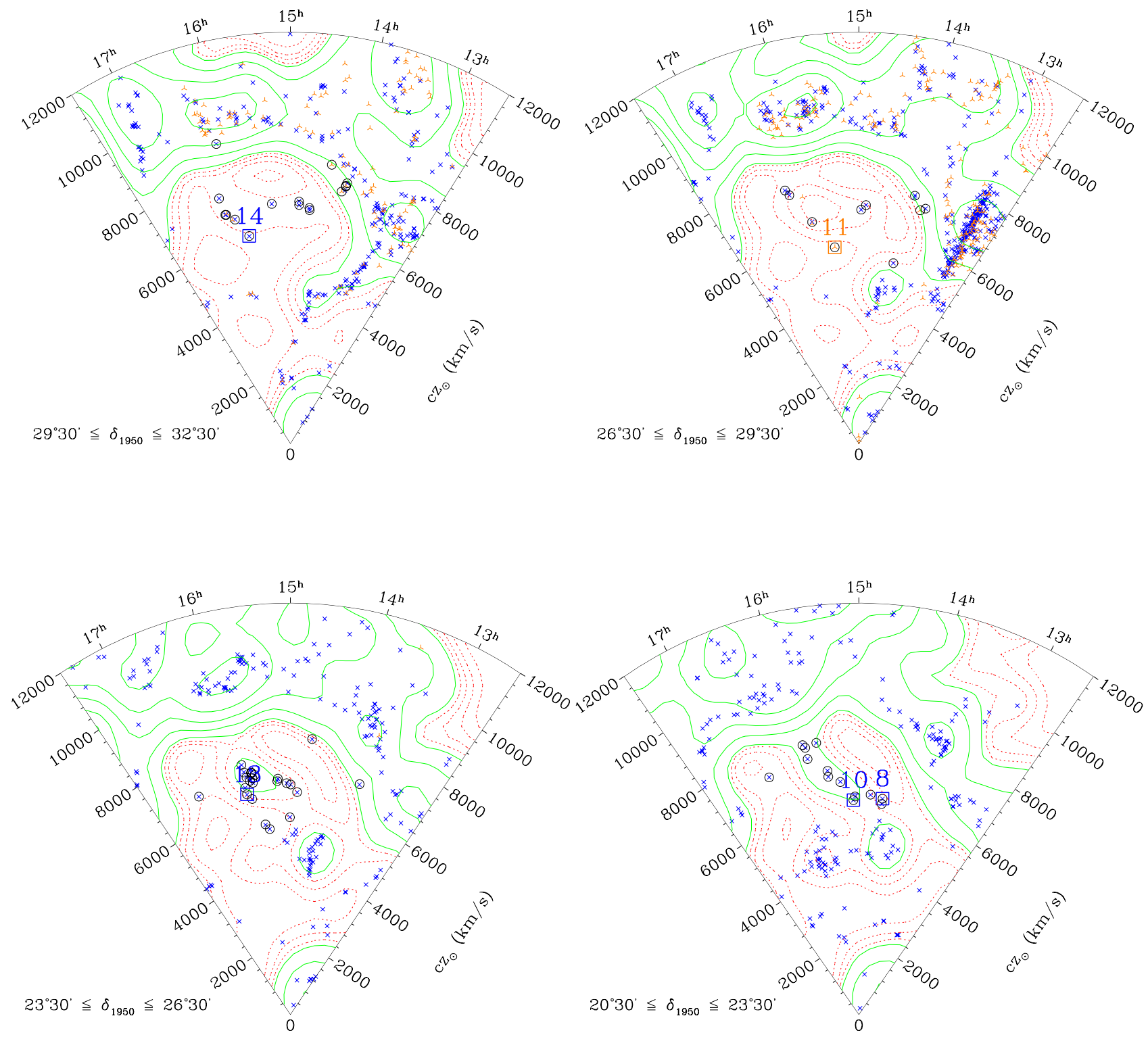
Fig. 2.- As Fig. 1, for CfA2 South voids.
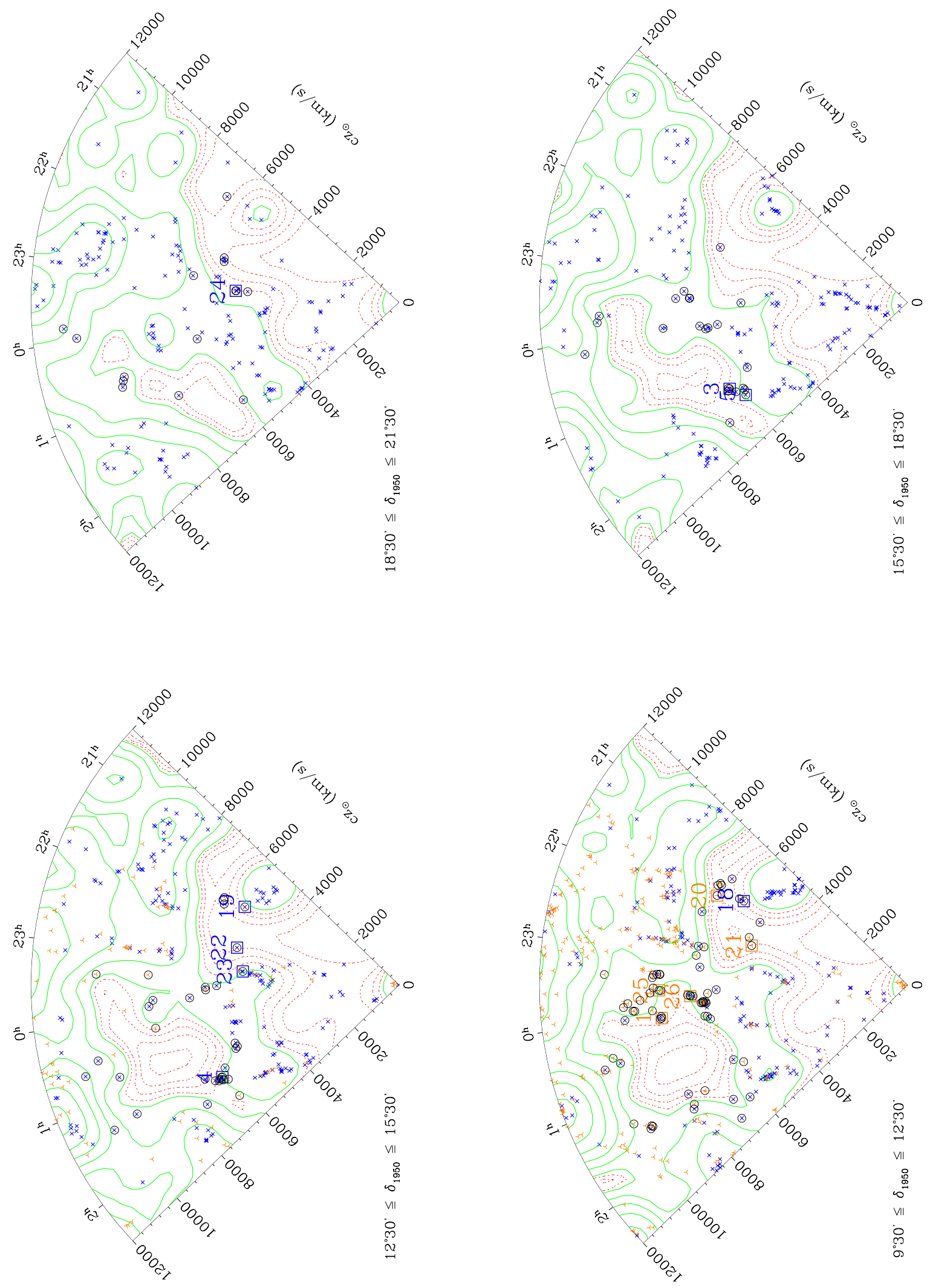
Fig. 2, - Cont'd.
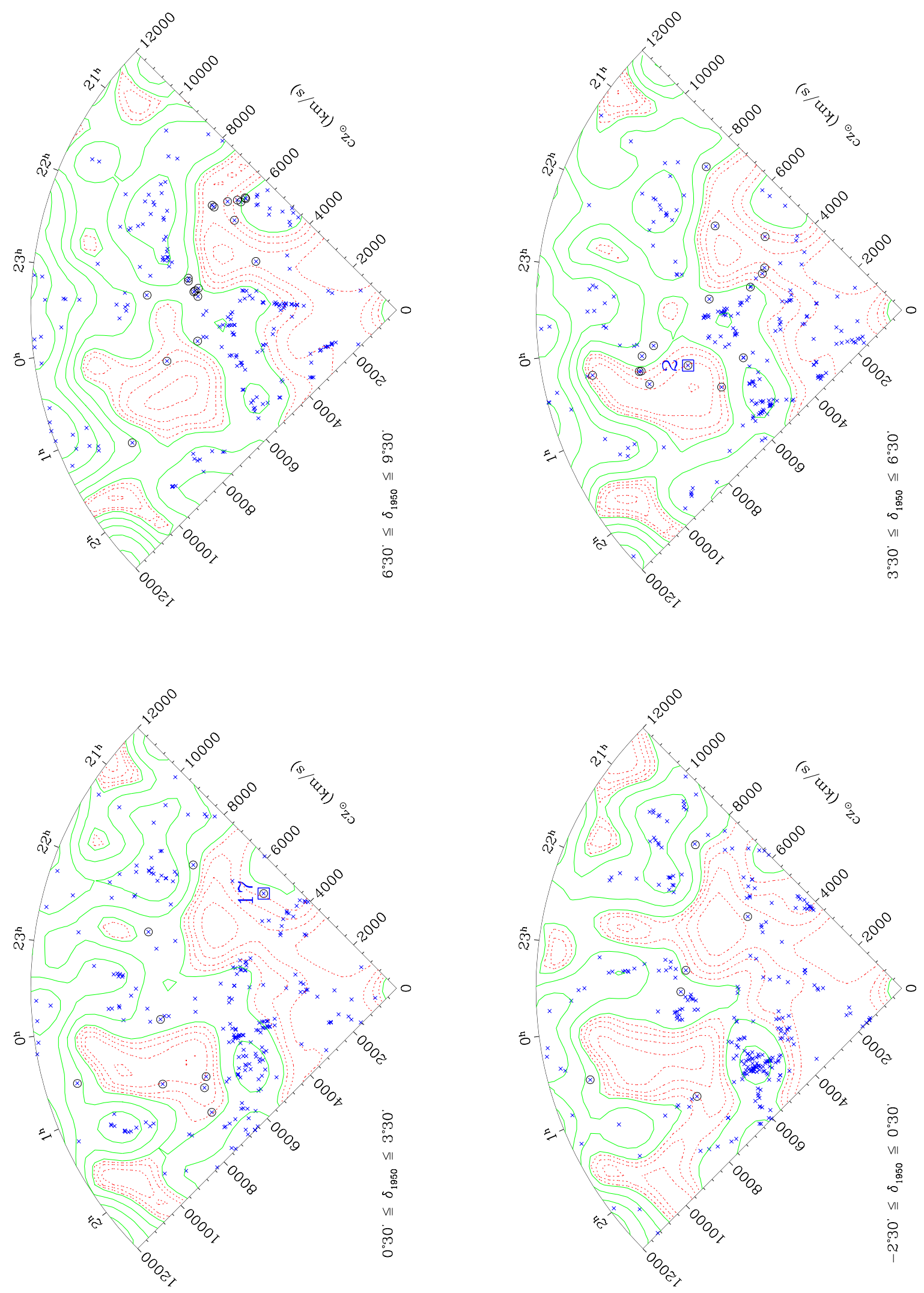

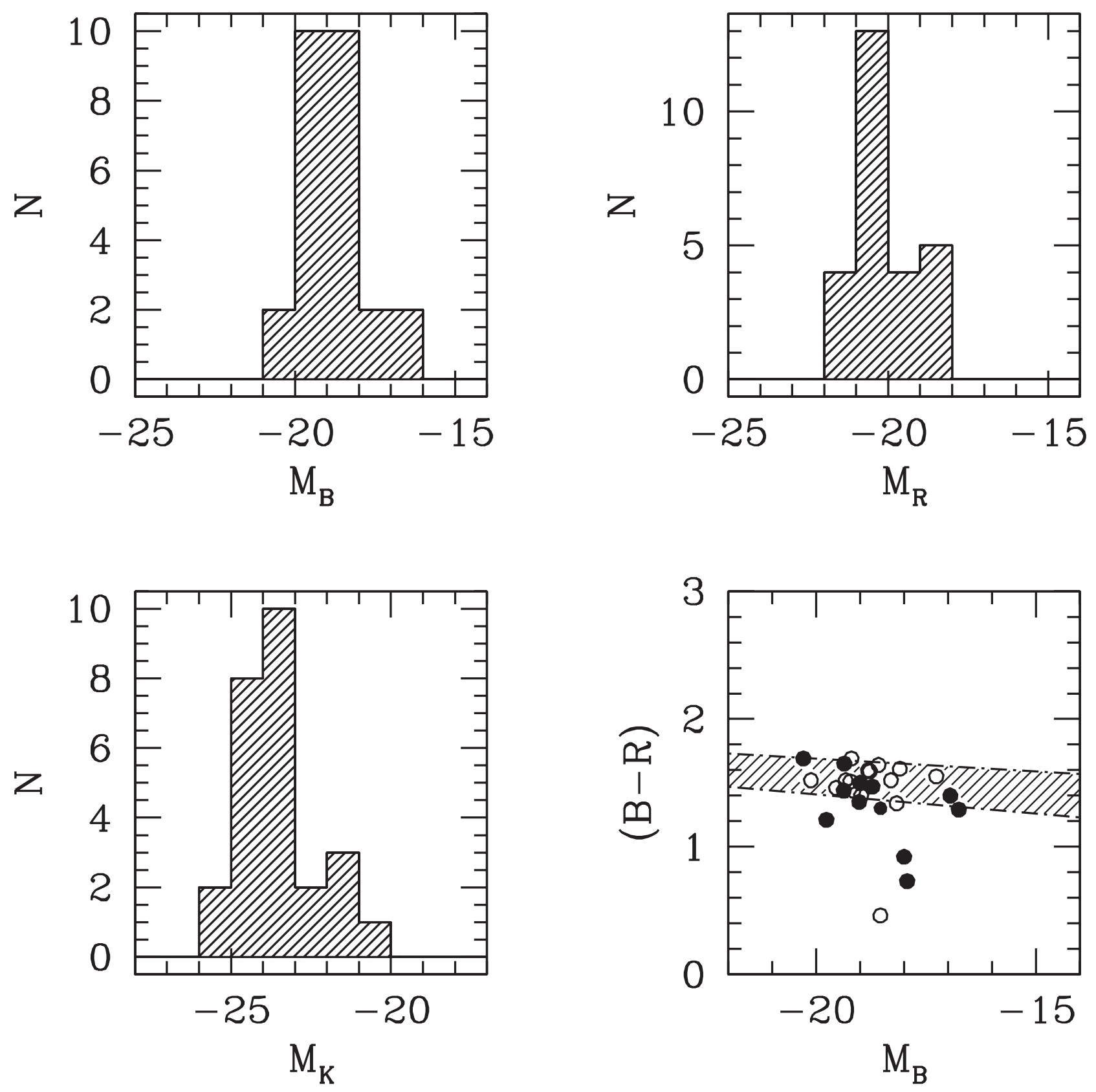

Fig. 3.- Histograms of the total $B, R$, and $K$ magnitudes of the void galaxies and the color magnitude diagram. Objects showing $\mathrm{H} \beta$ emission are shown as filled circles and the location of the red sequence in the Coma cluster estimated from Mobasher et al. (2001) is indicated. 


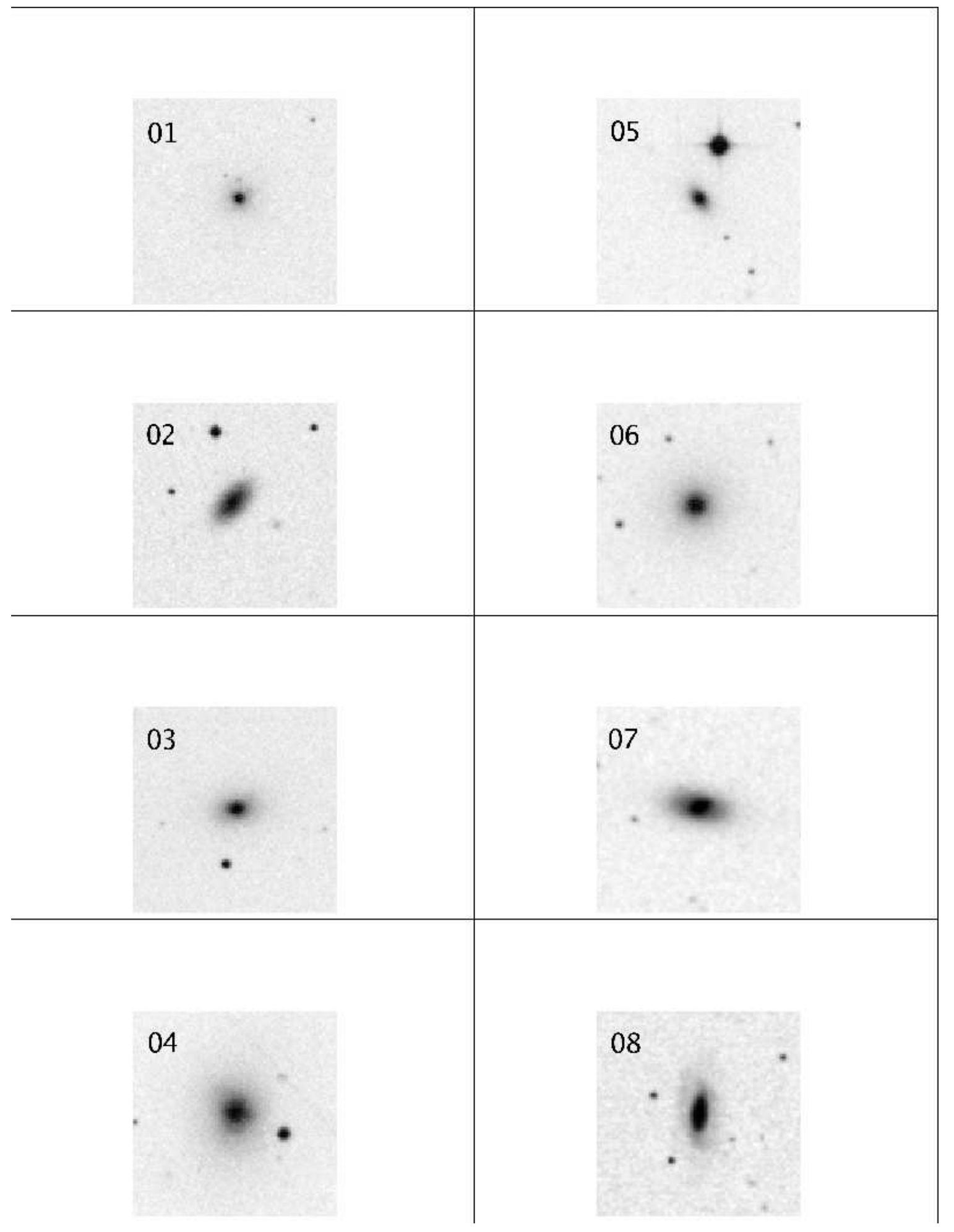

Fig. 4. - Images of the VGs from the digitized POSS2 blue data as referenced in the text. North is to the top and East is to the left. Each picture is $2^{\prime} X 2^{\prime}$ on a side. The compressed files of the "Palomar Observatory - Space Telescope Science Institute Digital Survey" of the northern sky, based on scans of the Second Palomar Sky Survey are copyright (C) 1993-1995 by the California Institute of Technology. 


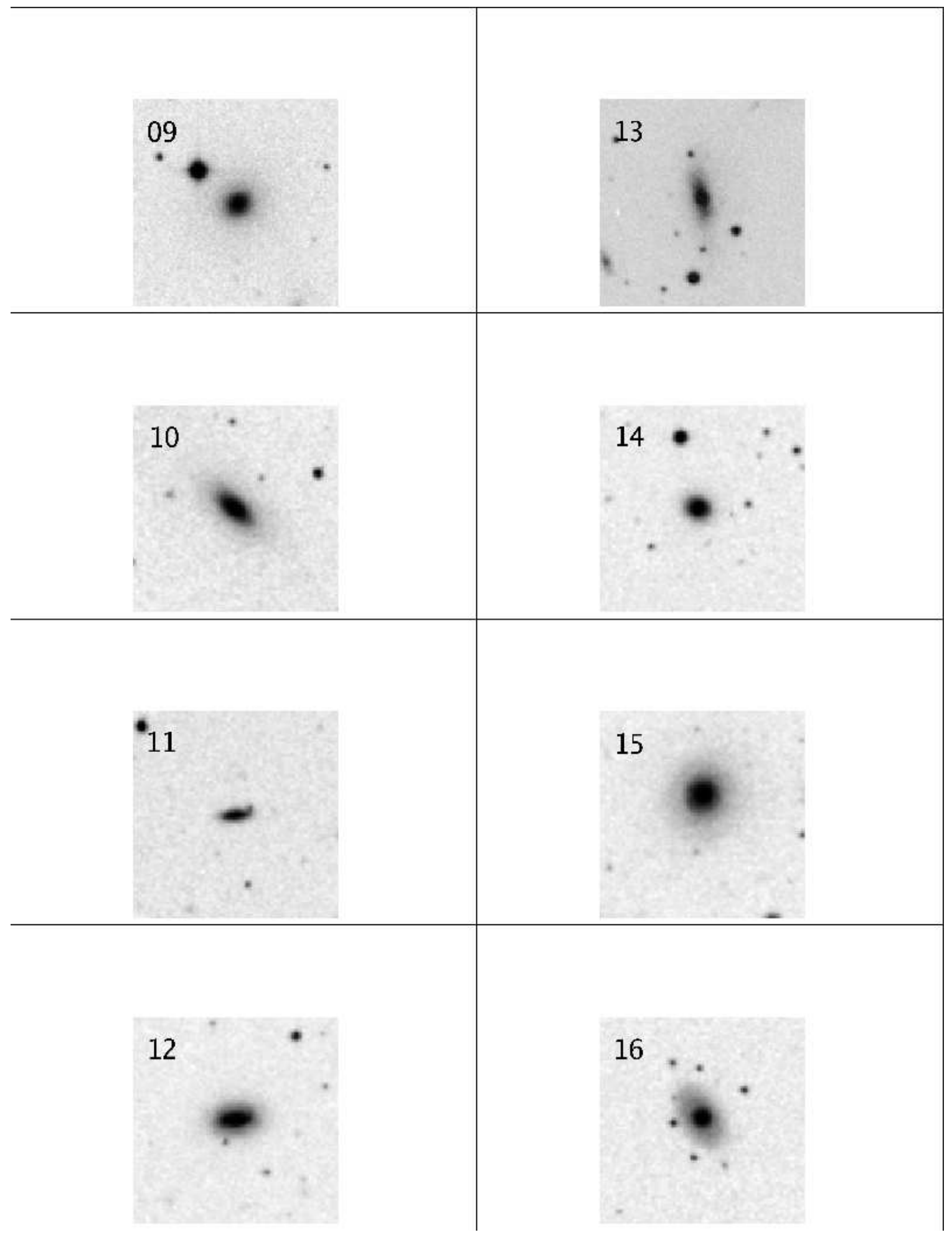

Fig. 4.- Cont'd. 


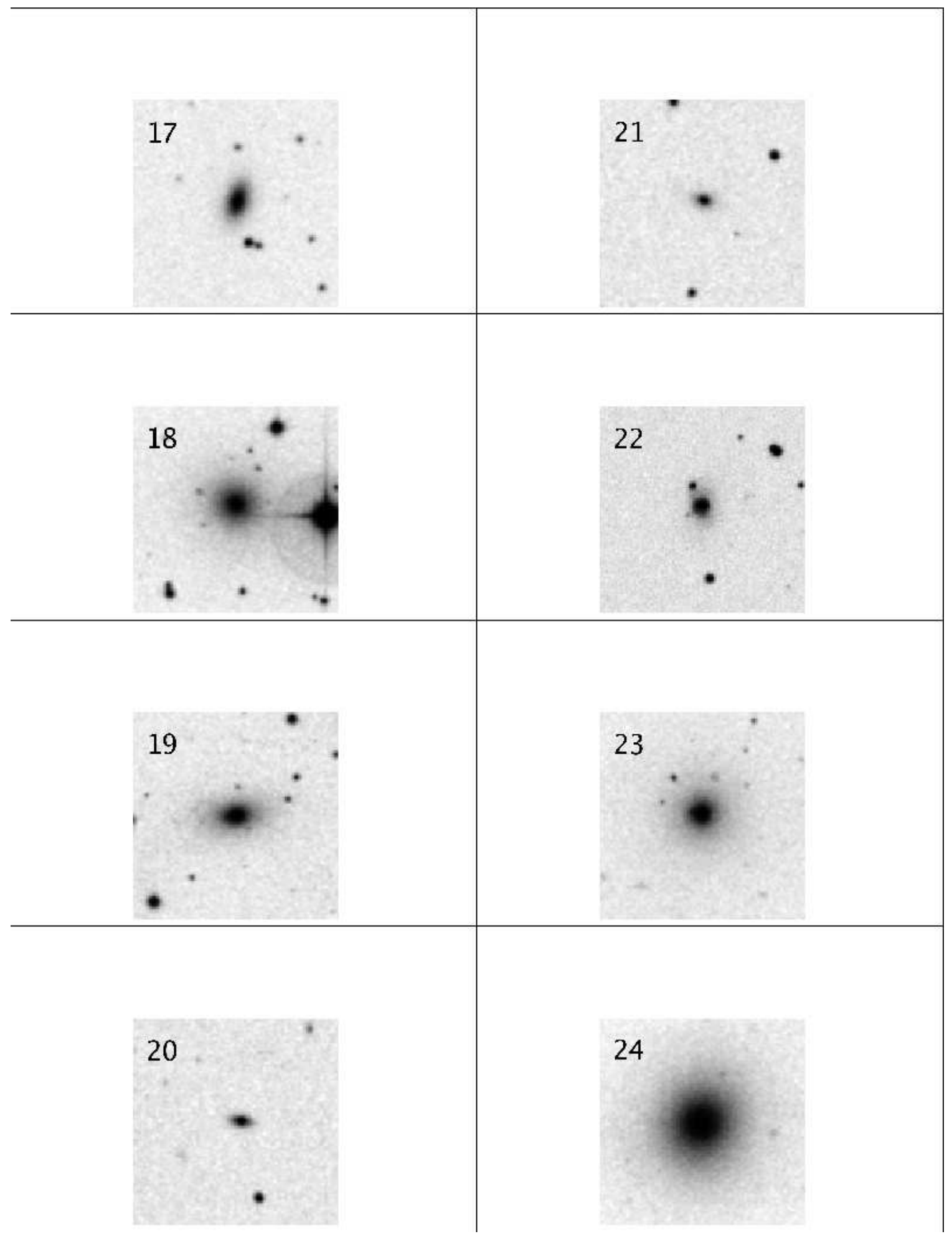

Fig. 4.- Cont'd. 


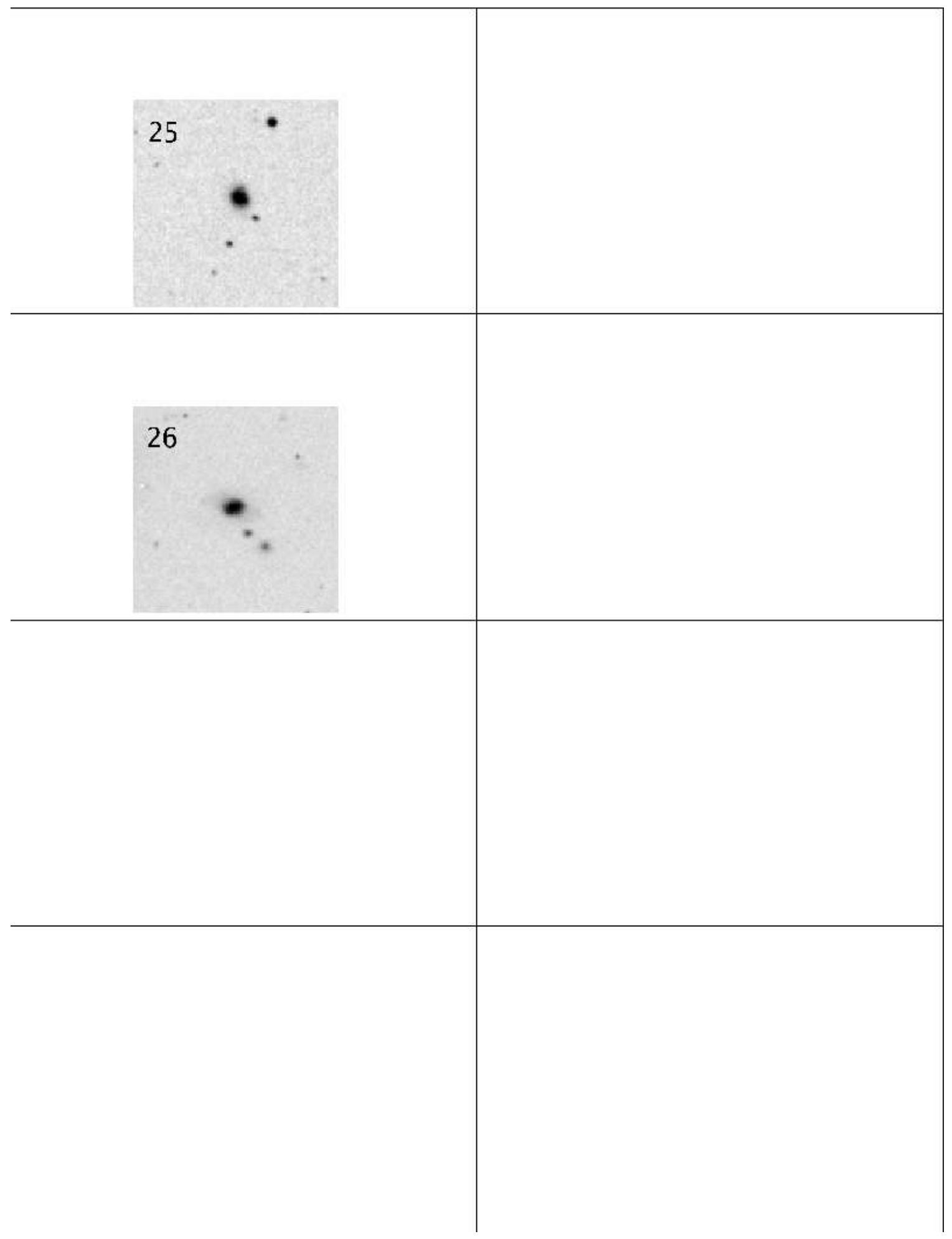

Fig. 4.- Cont'd. 
(4) IC1700

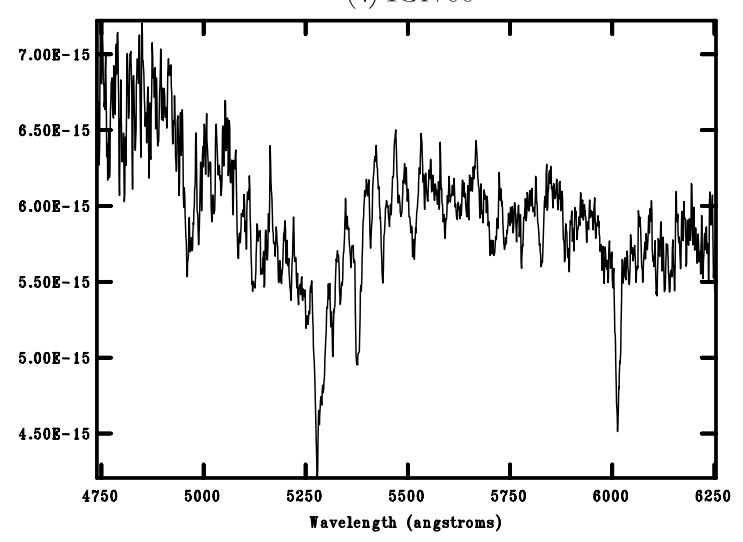

(15) $1600.6 \mathrm{p} 4302$

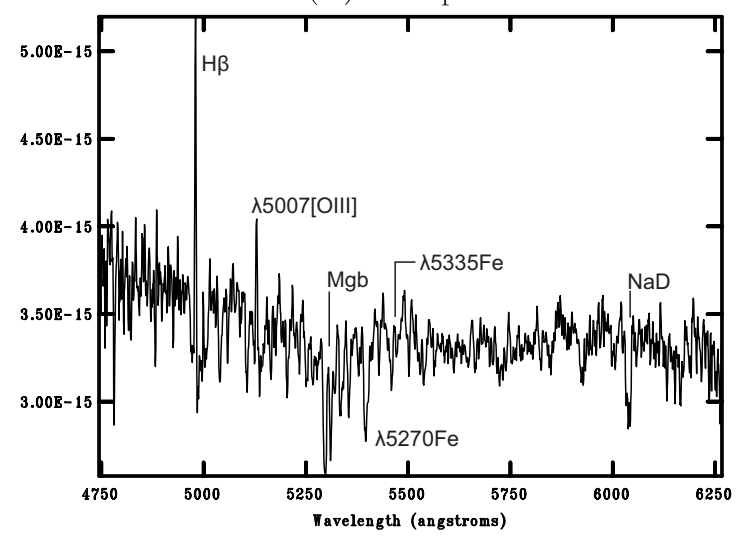

(17) $2106.7 \mathrm{p} 0318$

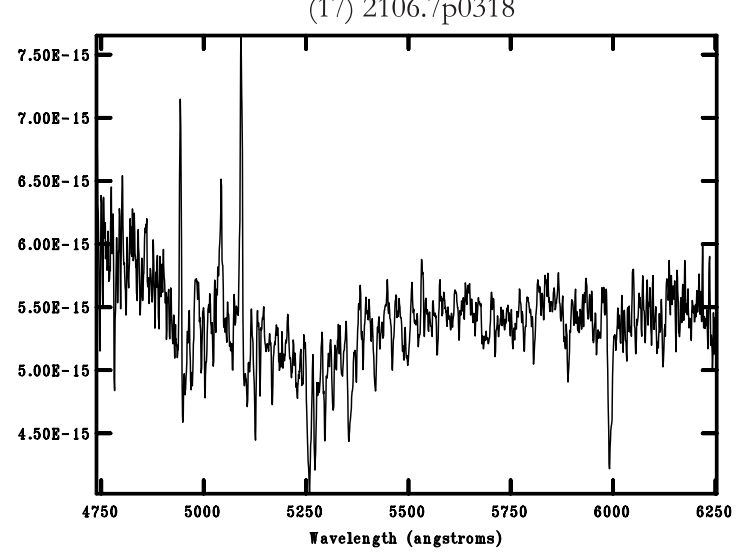

(4) IC1700

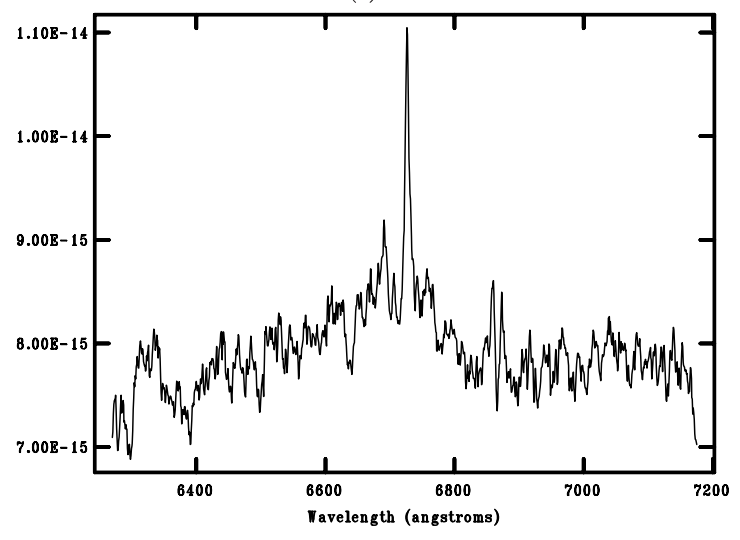

(15) $1600.6 \mathrm{p} 4302$

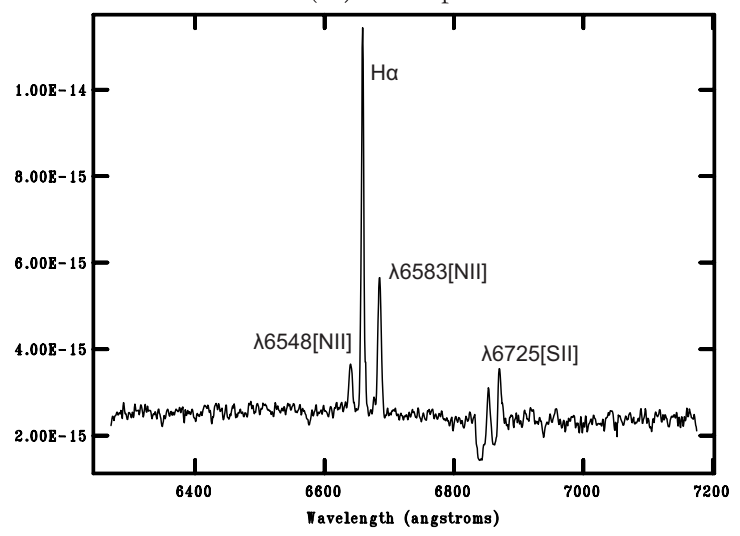

(17) $2106.7 \mathrm{p} 0318$

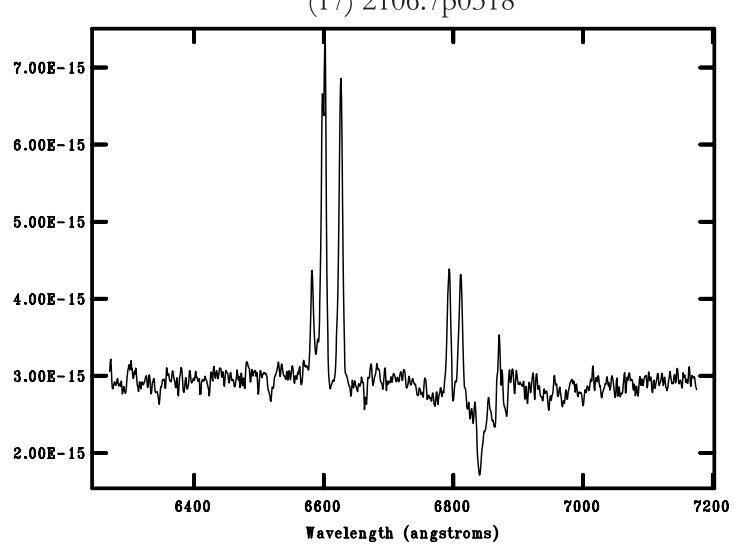

Fig. 5.- Examples of some of the VG spectra. Blue portion to the left and red to the right. The spectra have been flux corrected, but flux values are relative as not all objects were observed under photometric conditions. Some of the more important spectral features that occur in all of the spectra are indicated in the middle panels for the galaxy $1600.6 \mathrm{p} 4302$. 

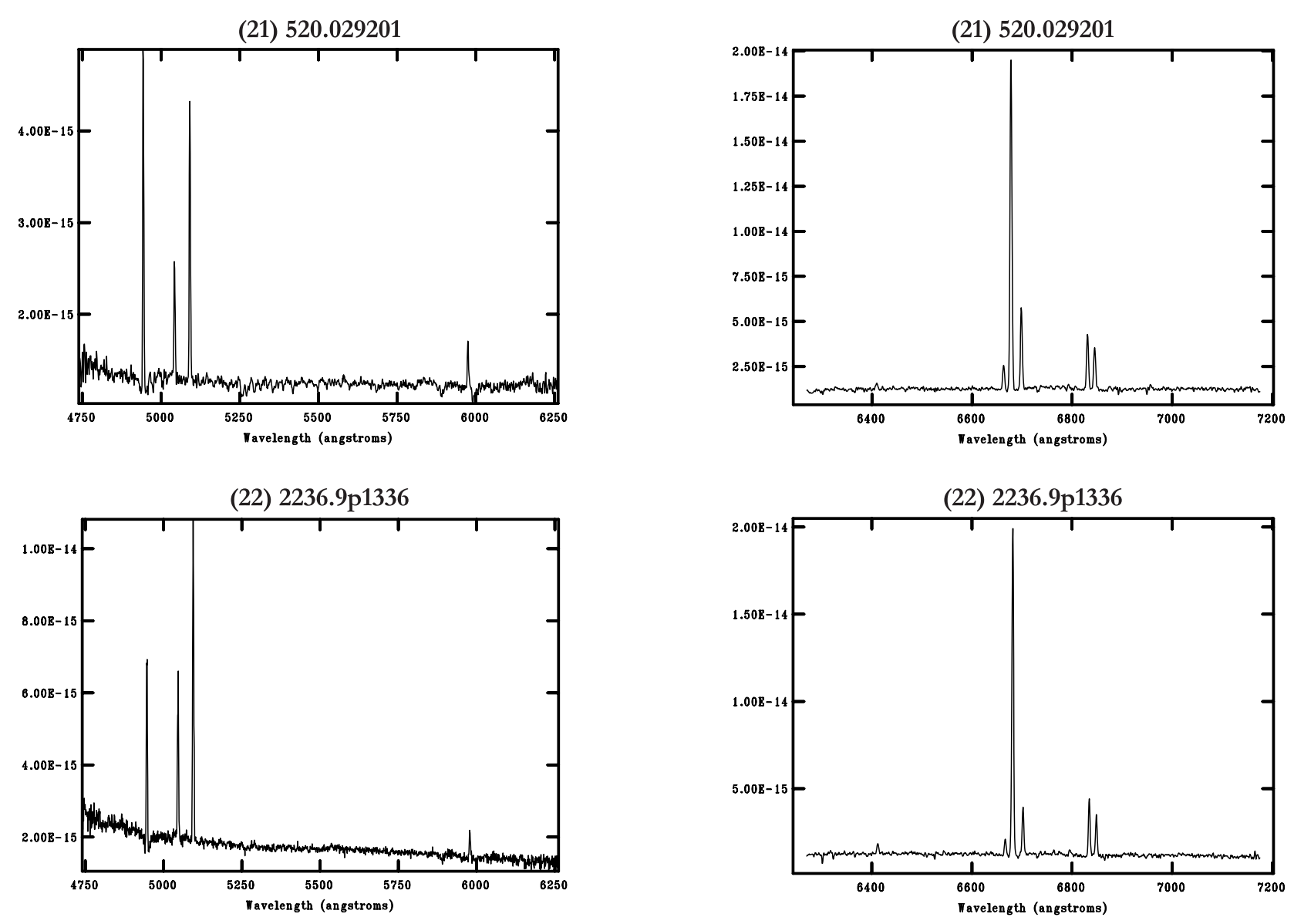

(24) NGC 7550
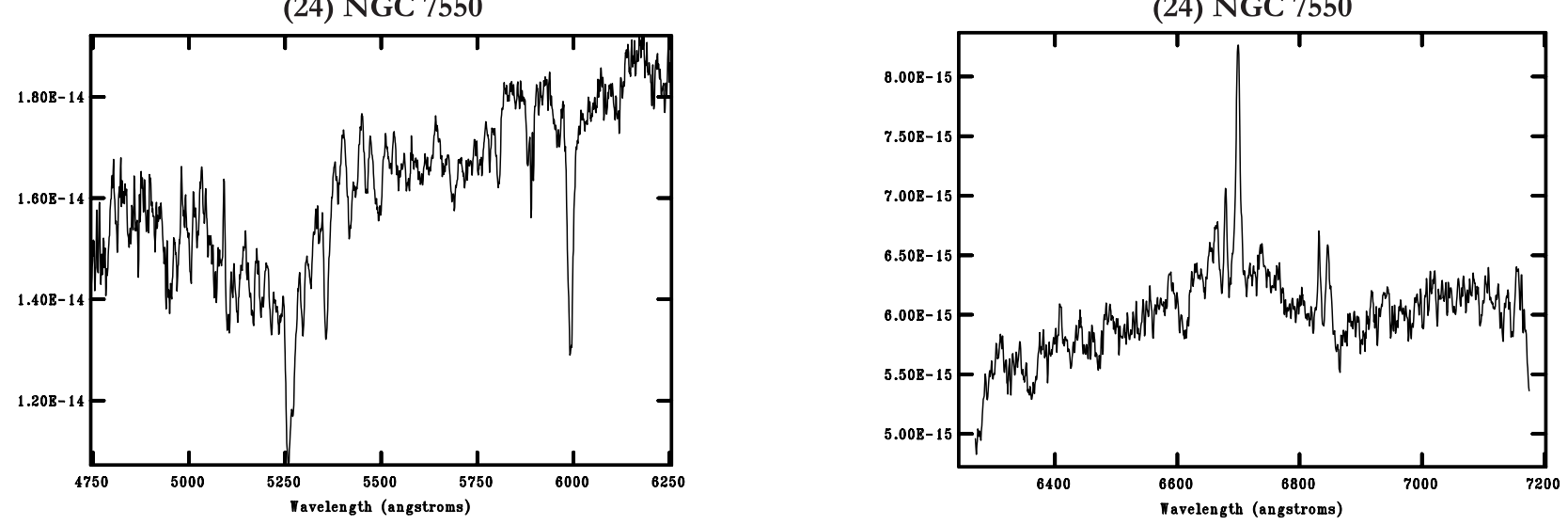

Fig. 5.- Cont'd. 

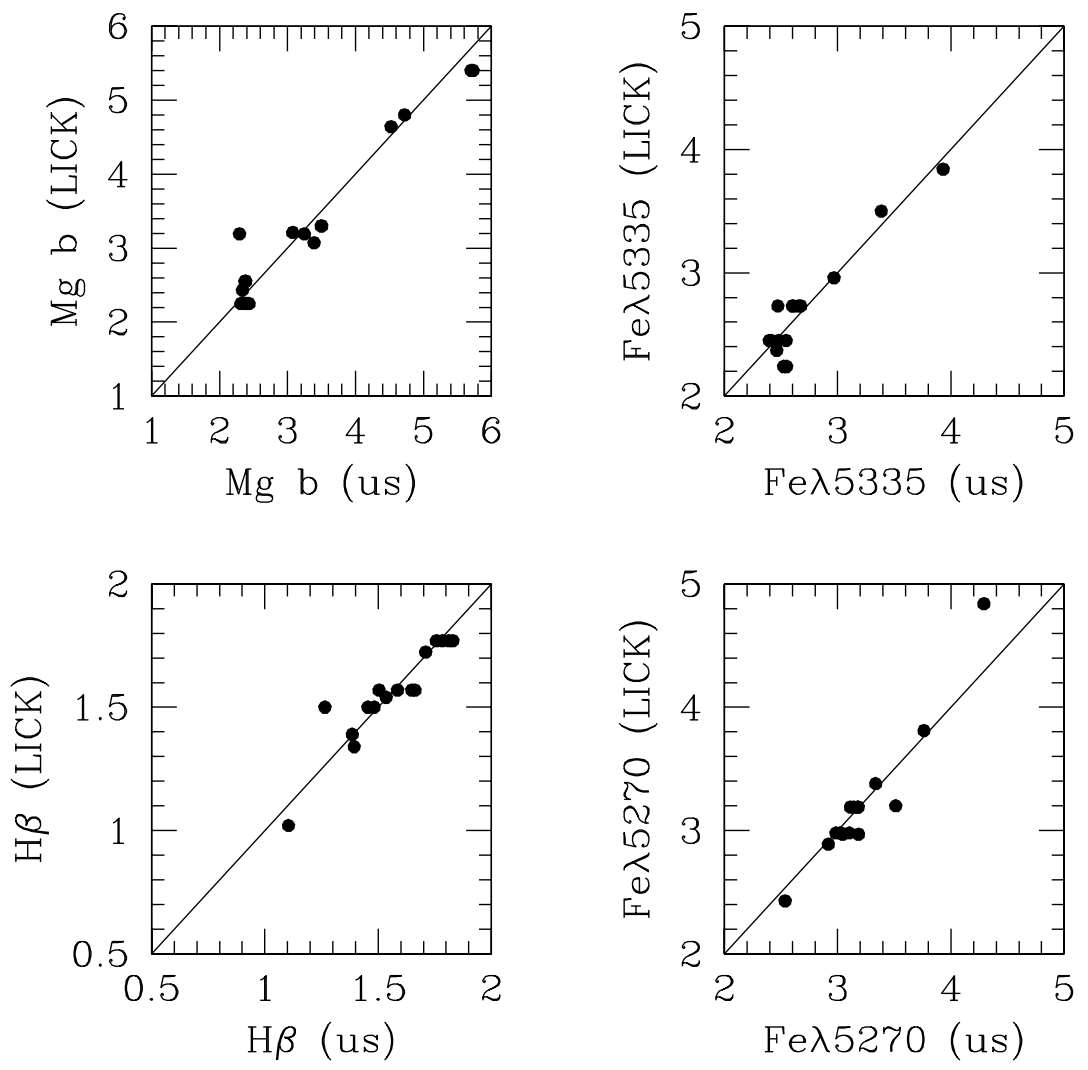

Fig. 6. - Comparison between the line indices measured in this study with the Lick indices in Worthey et al. (1994) and Trager et al. . (1998). 


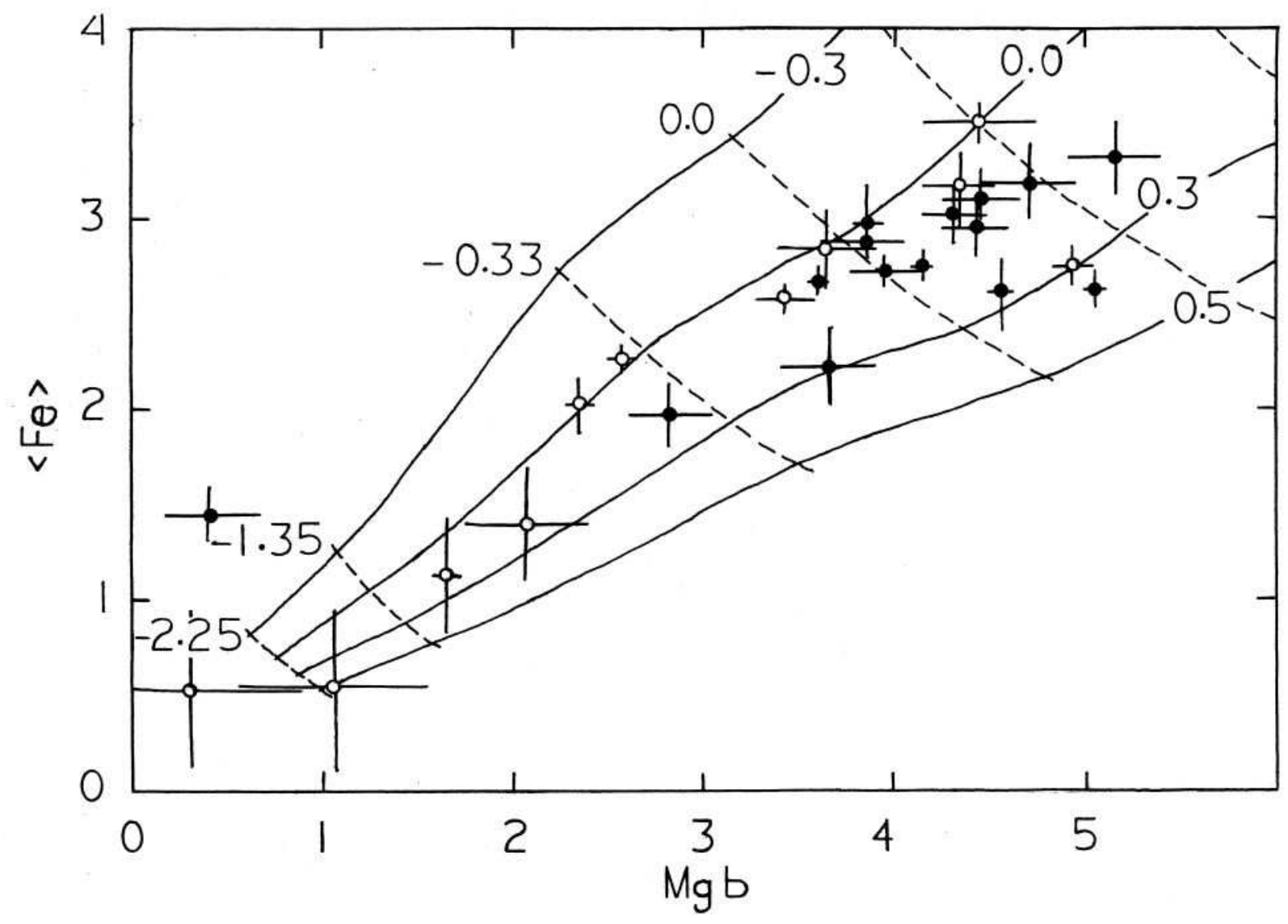

Fig. 7. - The positions of the observed galaxies with their error bars. The curves use the TMB models for an assumed age of 12 Gyr. Solid curves denote loci of constant $[\alpha / F e]$ and dashed curves indicate loci of fixed $[Z / H]$. Objects with detected $\mathrm{H} \beta$ emission are open circles. 


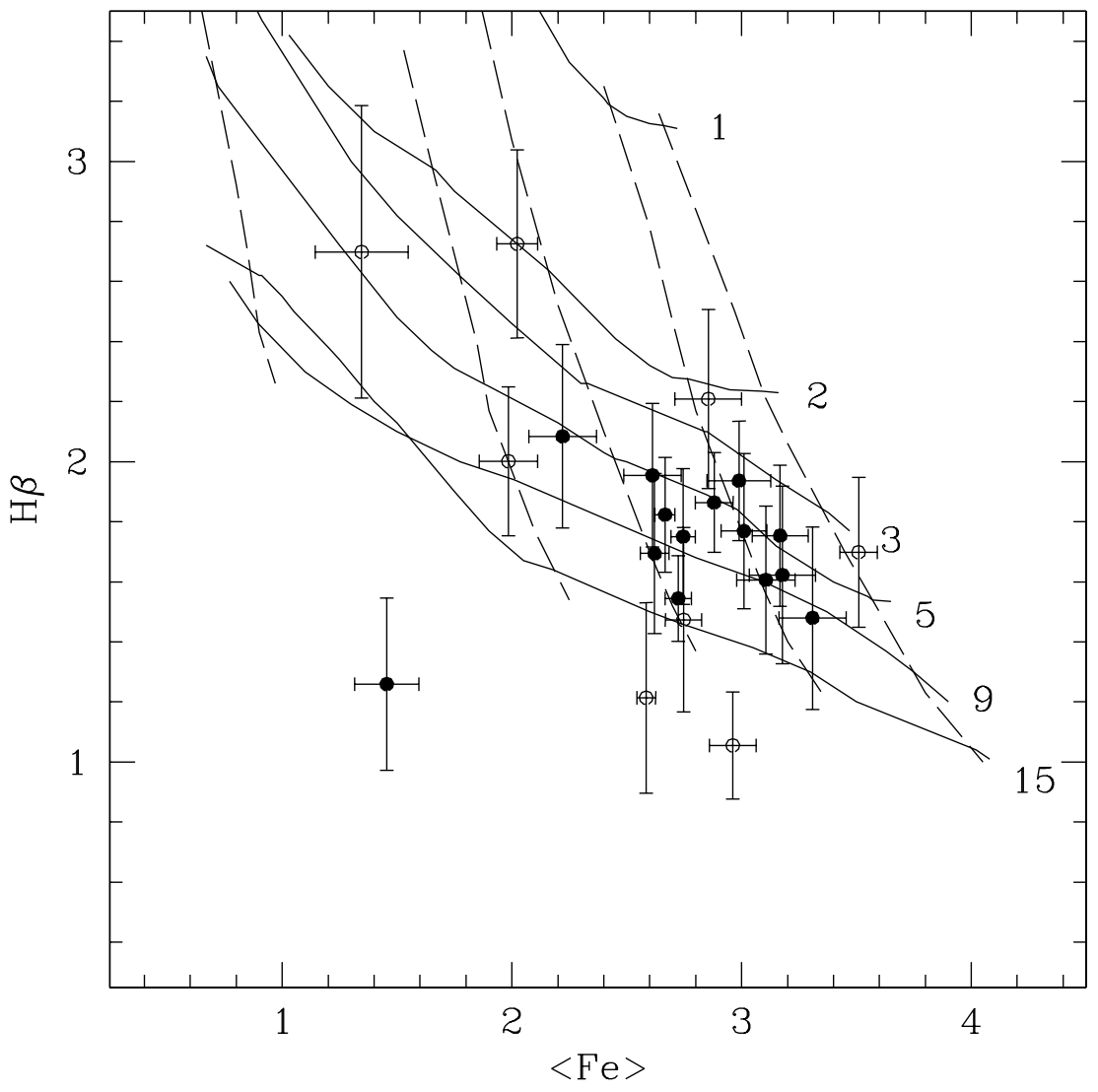

Fig. 8. - Positions of 22 VGs with their error bars. Shown are curves of constant age in Gyr (solid curves) and constant metal abundance, $[Z / H]$ (dashed curves: $[Z / H]=0.67,0.35,0.0,-0.33,-1.35$, from right to left, interpolated from TMB models for $[\alpha / H]=0.15$. Objects with detected $\mathrm{H} \beta$ emission are open circles. 

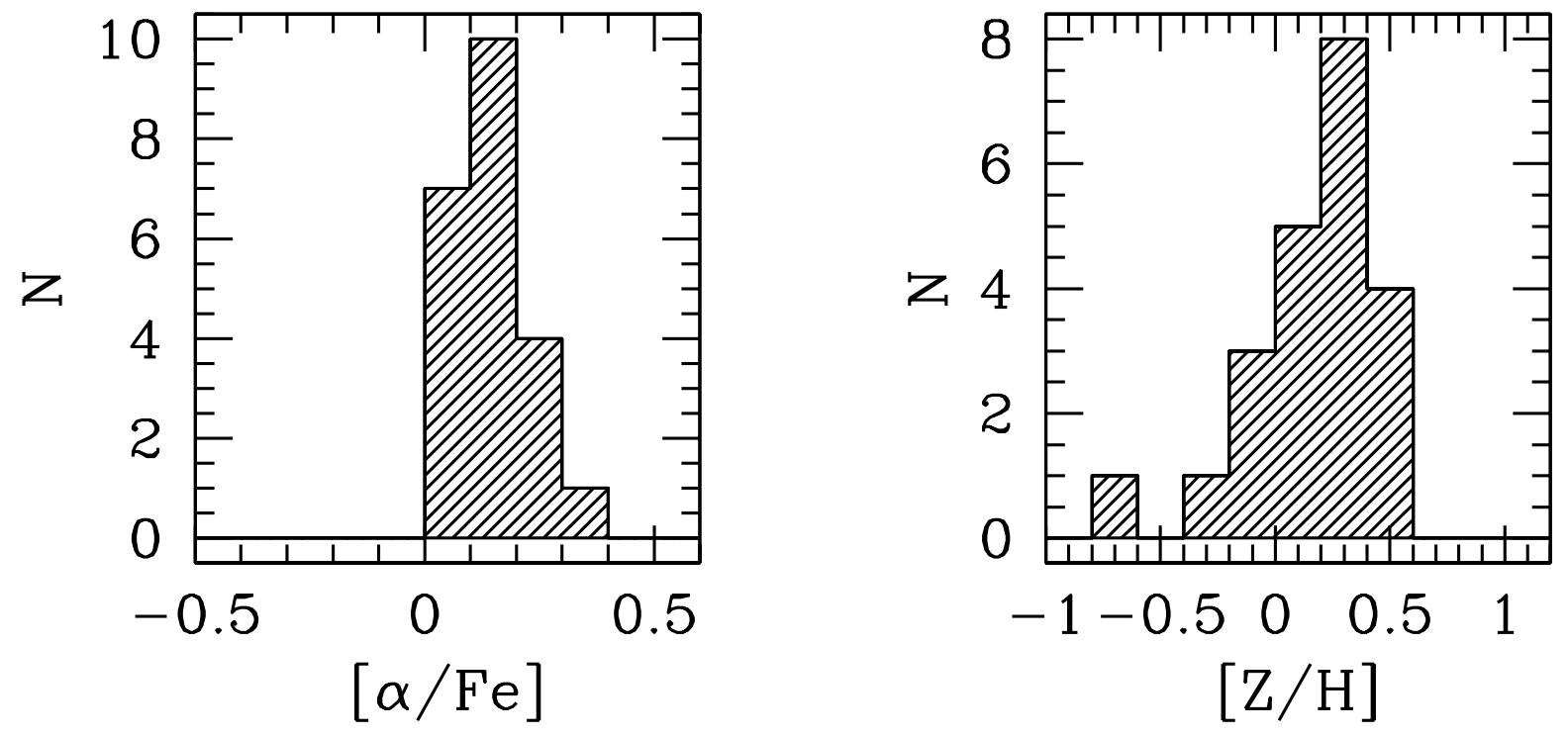

Fig. 9.- Histograms of $[Z / H]$ and $[\alpha / F e]$ for the program galaxies derived from $\left(\mathrm{Mg}_{b}\right.$ $<\mathrm{Fe}>$ ) diagrams using the TMB models and the derived ages of the objects as described in the text. 


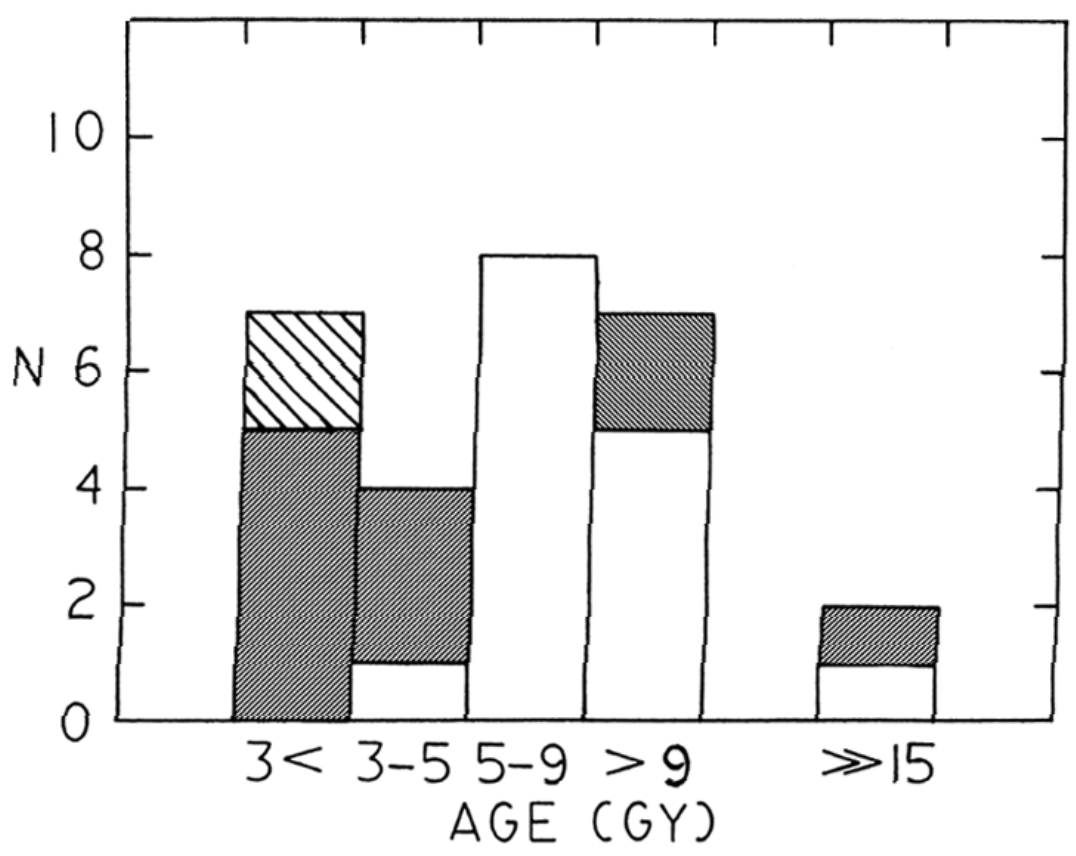

Fig. 10. - Histogram of the ages of galaxies read from Figure 8. Filled bars represent galaxies observed with $\mathrm{H} \beta$ emission. Open bars denote objects for which no $\mathrm{H} \beta$ emission component was detected. The $\gg 15$ column denotes objects that lie outside the grid in Figure 8 . They are added at the top of the $3<$ column as the hatched section following the discussion in the text. 

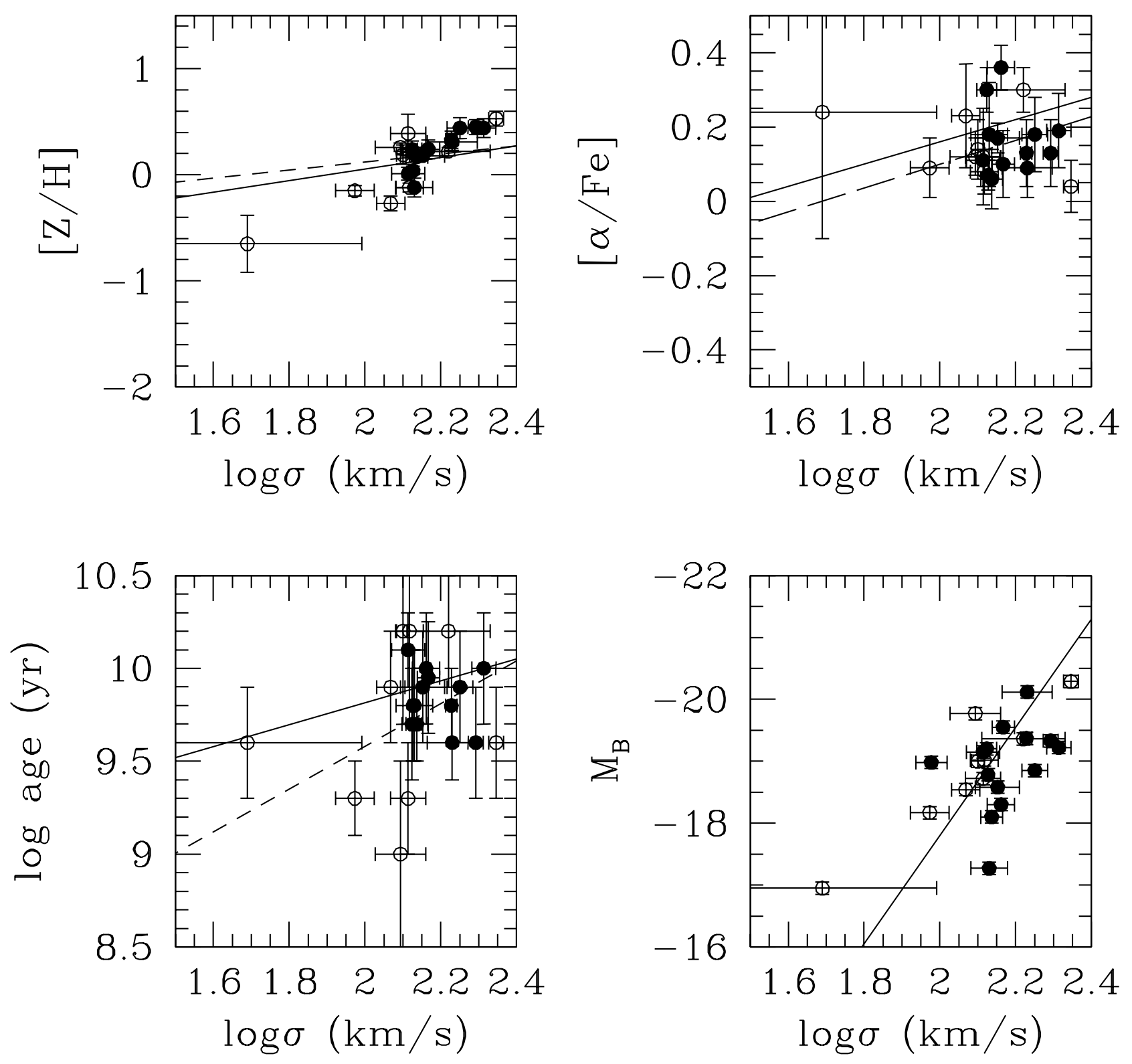

Fig. 11. - The metal abundances, ages, and absolute $B$ magnitudes of the VGs plotted against available velocity dispersions. The linear relations for the cluster sample of Nelan et al. (2005) are the solid lines and those of the Bernardi et al. (2006) study are the dashed. The line in the $\left(M_{B}, \log \sigma\right.$ plot is the Faber-Jackson relation of the Coma Cluster (Dressler et al. 1987). Objects with detected $\mathrm{H} \beta$ emission are open circles. 

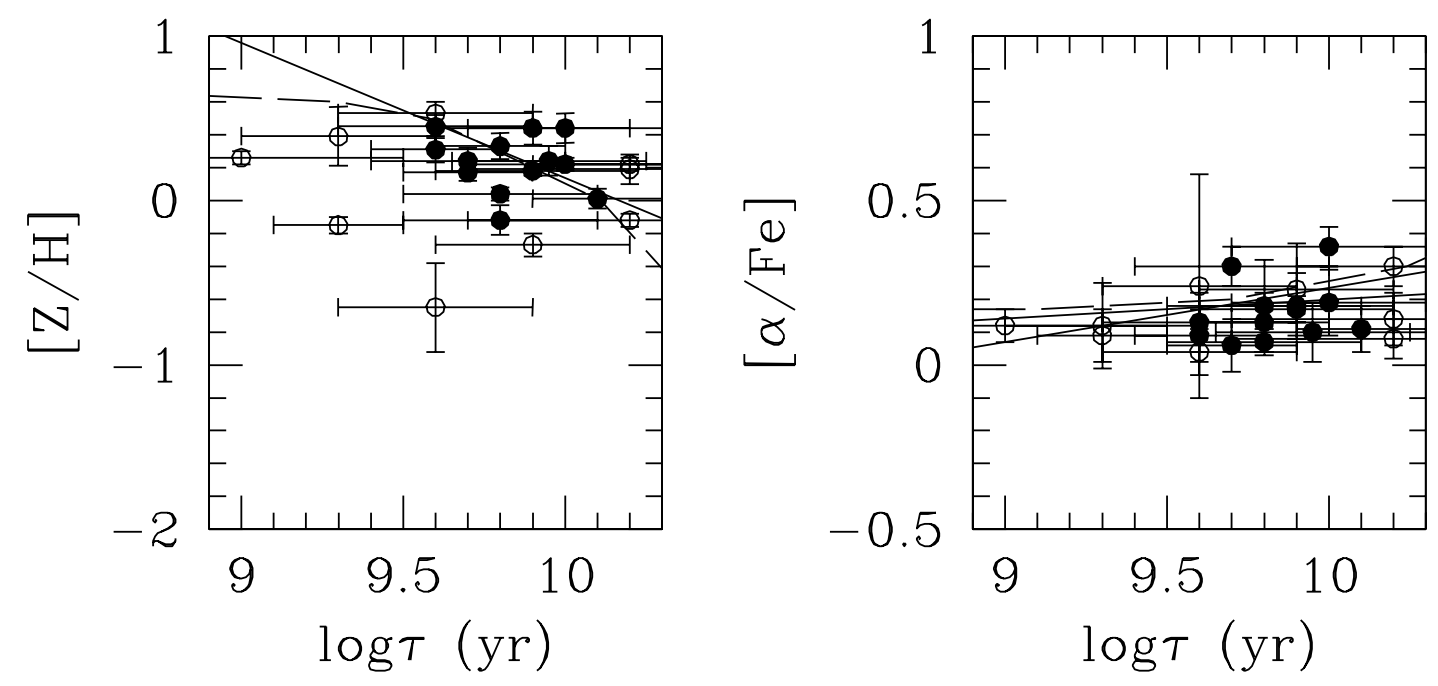

Fig. 12.- The metal abundances, $[\alpha / \mathrm{Fe}]$ and $[\mathrm{Z} / \mathrm{H}]$ plotted against logarithm of age, $\tau$ for galaxies with ages in Table 4. The solid lines are the relations given in Bernardi et al. (2006) and the dashed curves are from Mehlert et al. (2003) for the Coma cluster. Objects with detected $\mathrm{H} \beta$ emission are open circles. 


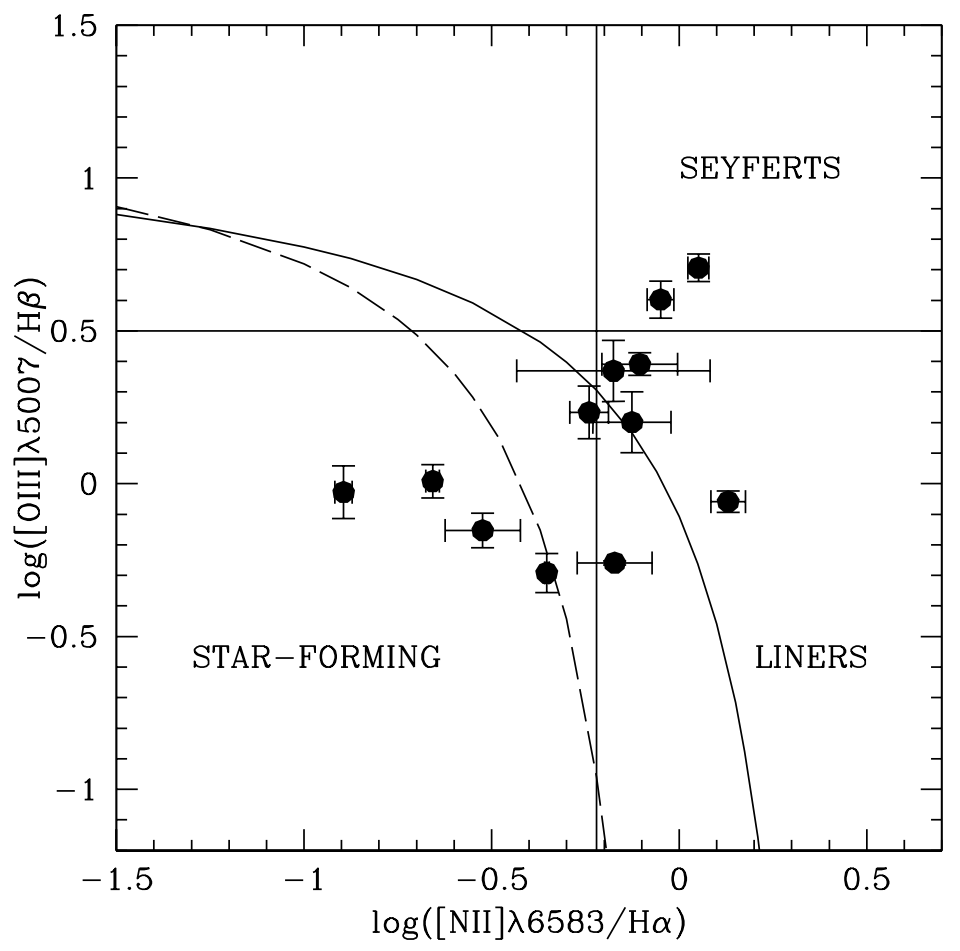

Fig. 13. - Diagnostic diagram showing the positions of the galaxies in Table 3 with error bars. The line separating the Seyfert and liner regions is that given in Yan et al. (2006). The solid and dashed curves demarcating the edge of the star-forming region are taken from Kewley et al. (2001) and Kaufmann et al. (2003). 